\title{
TEMPERED ENDOSCOPY FOR REAL GROUPS III: INVERSION OF TRANSFER AND L-PACKET STRUCTURE
}

\author{
D. SHELSTAD
}

\begin{abstract}
This paper examines adjoint relations for spectral analogues of the geometric transfer factors of Langlands and Shelstad in the case of the tempered spectrum of a real reductive algebraic group where the complex points are connected. Each tempered irreducible character is then expanded explicitly in terms of endoscopic characters. The analysis is also reinterpreted in terms of structure on $L$-packets in the form conjectured recently in much greater generality by Arthur. A triviality result is proved for the Whittaker normalization of spectral transfer factors which simplifies the results for certain inner forms of a quasi-split group.
\end{abstract}

\section{INTRODUCTION}

This is the last part of a project in which we use the canonical (geometric) transfer factors defined in [LS1] for all local fields of characteristic zero to prove stronger explicit versions of old results about tempered endoscopy, or $L$-indistinguishability, for real groups [S3]. We have introduced canonical spectral transfer factors as well in our setting [S1, S2], and completed a proof of the main transfer theorem directly in terms of these and the geometric factors. Our plan now is to invert the spectral identities of the transfer theorem in the same manner, and then to describe familiar pairings on tempered $L$-packets, but now explicitly in terms of transfer factors. Our formula for the pairing both satisfies Arthur's recent conjecture in A1 for the case of real $K$-groups and reconciles the implicitly defined formulation of [S3]. It also leads us naturally to an analysis of the Whittaker normalization of absolute transfer factors introduced first in $\underline{\mathrm{KS}}$ for the geometric side in the more general setting of twisted endoscopy over a local field of characteristic zero. We then prove the expected simpler statements for real $K$-groups of quasi-split type.

Inverting the spectral identities means that we write each tempered irreducible character on a real reductive group explicitly as a combination of endoscopic characters, i.e. as a combination of the virtual characters obtained by transfer of stable tempered characters on endoscopic groups. We will find that the weights in these combinations are the (adjoint) spectral transfer factors for which we have provided explicit formulas.

The inversion rests on explicit adjoint relations for our spectral transfer factors $\Delta_{\text {spec }}$ defined initially as a product $\Delta_{I} \Delta_{I I} \Delta_{I I I}$ in the $G$-regular case (see [S2]). These relations are motivated by Arthur's parallel relations for geometric factors in [A2], and we place the spectral factors in that setting, where several inner forms

Received by the editors January 15, 2008.

2000 Mathematics Subject Classification. Primary 22E45, 22E50.

(C)2008 American Mathematical Society Reverts to public domain 28 years from publication 
are handled simultaneously. The use of several inner forms is due originally to Vogan, but here we need the formulation of Kottwitz discussed in A2 . That turns out to be a useful setting for our construction of spectral $\Delta_{I I}$ in terms of Harish Chandra's distribution $\Theta_{\lambda}^{*}$ and generalized Weyl denominators, as well as for the term $\Delta_{I I I}$, for the transfer theorem itself, and then finally for the adjoint relations and inversion.

Write $\boldsymbol{\Delta}$ for the extended spectral transfer factor. Then the final inversion formula may be written as

$$
\operatorname{Tr} \boldsymbol{\pi}(\mathbf{f})=\frac{1}{n(\boldsymbol{\pi})\|\boldsymbol{\Delta}\|^{2}} \sum_{\mathbf{s}_{s c}} \overline{\boldsymbol{\Delta}\left(\boldsymbol{\pi}^{s}, \boldsymbol{\pi}\right)} S t-\operatorname{Tr} \boldsymbol{\pi}^{s}\left(\mathbf{f}^{s}\right) .
$$

We will explain the terms shortly. Because of properties of the transfer factors this sum may also be written more simply as

$$
\frac{1}{n(\boldsymbol{\pi})} \sum_{\mathbf{S}_{s c}} \boldsymbol{\Delta}\left(\boldsymbol{\pi}^{s}, \boldsymbol{\pi}\right)^{-1} S t-\operatorname{Tr} \boldsymbol{\pi}^{s}\left(\mathbf{f}^{s}\right) .
$$

We also note that we may always normalize transfer factors so that

$$
\boldsymbol{\Delta}\left(\boldsymbol{\pi}^{s}, \boldsymbol{\pi}\right)= \pm 1
$$

for all $\boldsymbol{\pi}$ (see Section 4 of $[\underline{\mathrm{S}}$ ), and that $n(\boldsymbol{\pi})$ is the cardinality of the $L$-packet of $\pi$.

In this formula we are given a tempered irreducible admissible representation $\boldsymbol{\pi}$ of the $K$-group $\mathbf{G}(\mathbb{R})$ associated to a real reductive group $G$, and $\mathbf{f}$ is a Schwartz function on $\mathbf{G}(\mathbb{R})$. The right side is an expansion of the character

$$
\mathbf{f} \rightarrow \operatorname{Tr} \boldsymbol{\pi}(\mathbf{f})
$$

of $\boldsymbol{\pi}$ as tempered distribution on $\mathbf{G}(\mathbb{R})$. Each distribution

$$
\mathbf{f} \rightarrow S t-\operatorname{Tr} \boldsymbol{\pi}^{s}\left(\mathbf{f}^{s}\right)
$$

in this expansion comes from the transfer theorem (see Theorem 6.2). It is the transfer of the tempered stable character $S t-\operatorname{Tr} \boldsymbol{\pi}^{s}$ on an endoscopic group $\mathbf{H}^{s}(\mathbb{R})$, and is a virtual character on $\mathbf{G}(\mathbb{R})$ composed of representations in the $L$-packet of $\pi$ with spectral transfer factors as weights.

To describe which endoscopic characters appear in the expansion, we choose a homomorphism

$$
\varphi: W_{\mathbb{R}} \rightarrow{ }^{L} G
$$

representing the Langlands parameter for $\pi$ and write $S_{\varphi}$ for the centralizer of the image of $\varphi$ in $G^{\vee}$, the complex dual group of $\mathbf{G}$, and $S_{\varphi}^{a d}$ for the image of $S_{\varphi}$ in the adjoint form $G_{a d}^{\vee}$ of $G^{\vee}$. The group $\mathbb{S}_{\varphi}^{a d}$ of components of $S_{\varphi}^{a d}$, a sum of groups of order two, counts the representations in the $L$-packet of $\boldsymbol{\pi}$. It was used in Section 5 of [S3] to establish structure conjectured by Langlands for the tempered $L$-packets attached to a single group $G$. Now, as well as working with $\mathbf{G}$ in place of $G$, we will follow Arthur's refinement in [A1 and consider the inverse image $S_{\varphi}^{s c}$ of $S_{\varphi}^{\text {ad }}$ in the simply-connected covering $G_{s c}^{\vee}$ of $G_{a d}^{\vee}$, as well as its group $\mathbb{S}_{\varphi}^{s c}$ of components. This is also motivated by the setting of [ABV] and $[\mathrm{AV}]$. If $s_{s c}$ is a semisimple element of $S_{\varphi}^{s c}$ we write $s$ for its image in $G^{\vee}$. We will see that the corresponding term in the inversion formula depends only on the image of $s_{s c}$ in $\mathbb{S}_{\varphi}^{a d}$, and then take the summation over $\mathbb{S}_{\varphi}^{a d}$. 
Before continuing on to the structure on $L$-packets, we recall briefly the structure of the spectral transfer factors. Assume that $\mathbf{H}_{1}$ is endoscopic for $\mathbf{G}$. For the inversion formula, we are interested in the complex conjugate $\overline{\boldsymbol{\Delta}\left(\boldsymbol{\pi}_{1}, \boldsymbol{\pi}\right)}$ of the spectral factor $\boldsymbol{\Delta}\left(\boldsymbol{\pi}_{1}, \boldsymbol{\pi}\right)$ of [S2]. It is defined for all tempered irreducible admissible representations $\boldsymbol{\pi}_{1}$ of $\mathbf{H}_{1}(\mathbb{R})$ with appropriate central character and all tempered irreducible admissible representations $\boldsymbol{\pi}$ of $\mathbf{G}(\mathbb{R})$.

We start with the $\mathbf{G}$-regular setting. By definition, $\boldsymbol{\Delta}\left(\boldsymbol{\pi}_{1}, \boldsymbol{\pi}\right)$ is nonzero exactly when the $\mathbf{G}$-regular pair $\left(\boldsymbol{\pi}_{1}, \boldsymbol{\pi}\right)$ is related, i.e., the Langlands parameter for $\boldsymbol{\pi}_{1}$ is $\mathbf{G}$-regular and maps to the parameter for $\boldsymbol{\pi}$.

We may prescribe the normalization of transfer factor $\Delta$ by the choice of $\Delta\left(\bar{\pi}_{1}, \overline{\boldsymbol{\pi}}\right)$ for a fixed G-regular related pair $\left(\overline{\boldsymbol{\pi}}_{1}, \overline{\boldsymbol{\pi}}\right)$ (see Section 11 of [S2]; the notation $\overline{\boldsymbol{\pi}}_{1}, \overline{\boldsymbol{\pi}}$ has nothing to do with complex conjugation). Then

$$
\Delta\left(\boldsymbol{\pi}_{1}, \boldsymbol{\pi}\right)=\boldsymbol{\Delta}\left(\overline{\boldsymbol{\pi}}_{1}, \overline{\boldsymbol{\pi}}\right) \boldsymbol{\Delta}\left(\boldsymbol{\pi}_{1}, \boldsymbol{\pi} ; \overline{\boldsymbol{\pi}}_{1}, \overline{\boldsymbol{\pi}}\right),
$$

where $\boldsymbol{\Delta}\left(\boldsymbol{\pi}_{1}, \boldsymbol{\pi} ; \overline{\boldsymbol{\pi}}_{1}, \overline{\boldsymbol{\pi}}\right)$ is the canonical spectral factor

$$
\frac{\Delta_{I}\left(\boldsymbol{\pi}_{1}, \boldsymbol{\pi}\right)}{\boldsymbol{\Delta}_{I}\left(\overline{\boldsymbol{\pi}}_{1}, \overline{\boldsymbol{\pi}}\right)} \frac{\boldsymbol{\Delta}_{I I}\left(\boldsymbol{\pi}_{1}, \boldsymbol{\pi}\right)}{\boldsymbol{\Delta}_{I I}\left(\overline{\boldsymbol{\pi}}_{1}, \overline{\boldsymbol{\pi}}\right)} \Delta_{I I I}\left(\boldsymbol{\pi}_{1}, \boldsymbol{\pi} ; \overline{\boldsymbol{\pi}}_{1}, \overline{\boldsymbol{\pi}}\right)
$$

constructed in [S2], and extended to $K$-groups below in Section 5 .

Since

$$
\Delta\left(\boldsymbol{\pi}_{1}, \boldsymbol{\pi} ; \overline{\boldsymbol{\pi}}_{1}, \overline{\boldsymbol{\pi}}\right)= \pm 1
$$

(see [S2], Theorem 11.1), $\overline{\boldsymbol{\Delta}\left(\boldsymbol{\pi}_{1}, \boldsymbol{\pi}\right)}$ is again a transfer factor $\boldsymbol{\Delta}^{\text {conj }}\left(\boldsymbol{\pi}_{1}, \boldsymbol{\pi}\right)$, but now normalized by the choice

$$
\boldsymbol{\Delta}^{\operatorname{conj}}\left(\overline{\boldsymbol{\pi}}_{1}, \overline{\boldsymbol{\pi}}\right)=\overline{\overline{\Delta\left(\overline{\boldsymbol{\pi}}_{1}, \overline{\boldsymbol{\pi}}\right)}}
$$

Moreover,

$$
\overline{\boldsymbol{\Delta}\left(\boldsymbol{\pi}_{1}, \boldsymbol{\pi}\right)}=\overline{\boldsymbol{\Delta}\left(\overline{\boldsymbol{\pi}}_{1}, \overline{\boldsymbol{\pi}}\right)} \frac{\boldsymbol{\Delta}_{I}\left(\boldsymbol{\pi}_{1}, \boldsymbol{\pi}\right)}{\boldsymbol{\Delta}_{I}\left(\overline{\boldsymbol{\pi}}_{1}, \overline{\boldsymbol{\pi}}\right)} \frac{\boldsymbol{\Delta}_{I I}\left(\boldsymbol{\pi}_{1}, \boldsymbol{\pi}\right)}{\boldsymbol{\Delta}_{I I}\left(\overline{\boldsymbol{\pi}}_{1}, \overline{\boldsymbol{\pi}}\right)} \boldsymbol{\Delta}_{I I I}\left(\boldsymbol{\pi}_{1}, \boldsymbol{\pi} ; \overline{\boldsymbol{\pi}}_{1}, \overline{\boldsymbol{\pi}}\right) .
$$

See Sections 8, 9 and 10 of $\left[\mathrm{S} 2\right.$ to recall the formula for $\boldsymbol{\Delta}_{I}$ (which parallels the first geometric term and involves the splitting invariant of a torus), for $\boldsymbol{\Delta}_{I I}$ (involving normalization of the stable Harish Chandra distribution $\Theta_{\lambda}^{*}$ ) and for $\boldsymbol{\Delta}_{I I I}$ (parallel to the third geometric term, now reflecting position in $L$-packet instead of position in stable conjugacy class). Recall also that $\boldsymbol{\Delta}_{I I}$ depends on the choice of $a$-data, $\boldsymbol{\Delta}_{I I I}$ on the choice of toral data, and that $\boldsymbol{\Delta}_{I}$ depends on both these choices, while the relative product $\boldsymbol{\Delta}\left(\boldsymbol{\pi}_{1}, \boldsymbol{\pi} ; \overline{\boldsymbol{\pi}}_{1}, \overline{\boldsymbol{\pi}}\right)$ is canonical.

If $\mathbf{G}$ is of quasi-split type we have the standard normalizations $\boldsymbol{\Delta}_{0}$ (since $\boldsymbol{\Delta}_{I I I}$ may then be written as a quotient also) and Whittaker normalizations $\boldsymbol{\Delta}_{\lambda}\left(\boldsymbol{\Delta}_{0}\right.$ modified by an epsilon factor) for the absolute factors. The factor $\boldsymbol{\Delta}_{0}$ depends on the choice of $\mathbb{R}$-splitting and $\boldsymbol{\Delta}_{\lambda}$ on the choice of Whittaker data $(B, \lambda)$, as on the geometric side [LS1, KS]. Also,

$$
\overline{\boldsymbol{\Delta}_{0}\left(\boldsymbol{\pi}_{1}, \boldsymbol{\pi}\right)}=\boldsymbol{\Delta}_{I}\left(\boldsymbol{\pi}_{1}, \boldsymbol{\pi}\right) \overline{\boldsymbol{\Delta}_{I I}\left(\boldsymbol{\pi}_{1}, \boldsymbol{\pi}\right)} \boldsymbol{\Delta}_{I I I}\left(\boldsymbol{\pi}_{1}, \boldsymbol{\pi}\right)
$$

(see Section 11 of [S2]), while

$$
\overline{\boldsymbol{\Delta}_{\lambda}\left(\boldsymbol{\pi}_{1}, \boldsymbol{\pi}\right)}=\boldsymbol{\Delta}_{\lambda}\left(\boldsymbol{\pi}_{1}, \boldsymbol{\pi}\right)= \pm 1
$$

([S2, Lemma 11.4), so that we get the simplest form of the inversion by using a Whittaker normalization. Here again we use the extension to $K$-groups below in Section 5. 
So far, we have described the spectral transfer factors for G-regular pairs $\left(\boldsymbol{\pi}_{1}, \boldsymbol{\pi}\right)$. That will be sufficient for the inversion in the case that $\boldsymbol{\pi}$ has regular parameter. In general, the spectral transfer factors are defined by (nondegenerate) coherent continuation, and so we need to show that this continuation fits naturally with our inversion method. The main step, adapted from [S3] to our present setting, is in Section 9. We also follow Section 5.4 of [S3], using a uniform version of the KnappZuckerman classification of the tempered spectrum, now for reductive $K$-groups, to see that endoscopic transfer captures all tempered characters on $\mathbf{G}(\mathbb{R})$.

As we will explain in more detail in Section 7, the inversion itself rests very simply on Theorem 7.5 which recovers and supplements results of Section 5 of S3. in the setting of $K$-groups. The theorem is proved in Sections 8, 9 and 10.

We have chosen the inversion problem as our primary focus only because it is easy to motivate in terms of building all tempered characters from the transfer of stable characters on endoscopic groups. Theorem 7.5 has another interpretation coming from stabilization of the Arthur-Selberg trace formula and global multiplicity formulas. This second interpretation of the theorem, which relates transfer factors to pairings on tempered $L$-packets, is in many ways more useful as a motivation for the broader questions for real groups discussed in $\mathrm{A}$. That applies, in particular, to interpreting the Adams-Barbasch-Vogan proof of the Arthur conjectures [ABV] (see Section 8 of $[\mathrm{A}]$ ). See also the discussion in [AJ, p. 274] on the failure of inversion in the earlier work of Adams-Johnson on transfer of cohomological representations.

Arthur has given some precise requirements for the pairings on tempered $L$ packets in the case of groups over nonarchimedean fields A1. We will observe in Corollary 11.2 that our formulation satisfies the requirements of Arthur's conjecture in the setting of real $K$-groups. The pairing is specified by factoring the spectral transfer factors of $[S 2$. Once we fix a base point for an $L$-packet and decide how it is to pair within Arthur's constraint, the rest of the pairing on the packet is uniquely determined: see Corollary 11.1 and Remark 11.3. In particular, we see concretely how the pairing may yield more than just a sign. At the same time we obtain intrinsic normalizations of all endoscopic characters (Lemma 11.4).

For a $K$-group of quasi-split type, there is a natural way to arrange that the pairings involve only signs: Whittaker normalization $\boldsymbol{\Delta}_{\lambda}=\varepsilon \boldsymbol{\Delta}_{0}$ of the absolute transfer factors. Then no factoring is needed. We first satisfy Arthur's constraint by choosing the attached generic representation as base point and pairing it as the trivial character. Then Theorem 11.5 will imply that

$$
\left\langle s_{s c}, \boldsymbol{\pi}\right\rangle=\boldsymbol{\Delta}_{\lambda}\left(\boldsymbol{\pi}^{s}, \boldsymbol{\pi}\right),
$$

for all semisimple $s_{s c}$ in $S_{\varphi}^{s c}$ and $\boldsymbol{\pi}$ in the $L$-packet $\boldsymbol{\Pi}$ attached to $\varphi$. The dependence on $s_{s c}$ and $s$ is through their common image in $\mathbb{S}_{\varphi}^{a d}$, and so $\boldsymbol{\Delta}_{\lambda}$ carries with it a dual pairing of $\boldsymbol{\Pi}$ with $\mathbb{S}_{\varphi}^{a d}$. This pairing is determined uniquely by the conjugacy class of $(B, \lambda)$. It may involve all inner forms (for example, $\mathbf{G}=\mathbf{S U}(n+1, n)$ ), the quasi-split form alone $\left(\mathbf{G}=\mathbf{S p}_{2 n}\right)$, or multiple copies of some nonquasi-split forms along with the quasi-split form $(\mathbf{G}=\mathbf{S U}(n, n))$.

It remains to prove Theorem 11.5 , that if tempered $\boldsymbol{\pi}$ is generic relative to $(B, \lambda)$, then

$$
\boldsymbol{\Delta}_{\lambda}\left(\boldsymbol{\pi}^{s}, \boldsymbol{\pi}\right)=1
$$

for all semisimple $s_{s c}$ in $S_{\varphi}^{s c}$. We may restrict our attention to the quasi-split component $G$ of $\mathbf{G}$ (see Lemma 12.6). Recall that $\Delta_{\lambda}\left(\pi^{s}, \pi\right)$ is defined initially for 
$G$-regular pairs $\left(\pi^{s}, \pi\right)$ as a product of four terms, two fourth roots of unity and two signs. Our proof in Section 13 avoids direct calculation of $\Delta_{\lambda}\left(\pi^{s}, \pi\right)$ except for the representations of the next paragraph, and even then we reduce to the familiar computation for $S L(2)$. Those representations allow us to handle discrete series representations when the derived group of $G$ is simply-connected. The rest of the argument relies on general properties of endoscopic transfer, and of course the entire discussion rests on results of Kostant $[\mathrm{K}]$ and Vogan $[\mathrm{V}]$ classifying generic representations of real groups.

In Section 12 we pause to gather various remarks. These concern mainly a family of tempered representations that generalize those with zero infinitesimal character discussed by Carayol and Knapp [CK]. The representations exist for each cuspidal reductive quasi-split group with simply-connected derived group and for its elliptic endoscopic groups. Their (small) $L$-packets consist entirely of generic representations, one for each $G(\mathbb{R})$-conjugacy class of Whittaker data. On the other hand, the representations are identified in such a way (with totally degenerate data) that the spectral transfer factors are not immediately evident. We observe another natural candidate for the attached pairing which we show to be correct in Lemma 12.1. Its definition is, unsurprisingly, analogous to that on the geometric side for regular unipotent conjugacy classes in Section 5 of [LS1. We note in passing that endoscopic inversion expresses the character of each representation very simply in terms of the stable versions of these same characters on endoscopic groups. Use of the appropriate Whittaker normalization removes the last extraneous constant.

See [S7] for some worked examples related to $K$-groups and spectral transfer factors.

Throughout this paper we delete the term $\Delta_{I V}$ from the geometric transfer factors $\Delta_{\text {geom }}$ and compatibility factors $\Delta_{\text {comp }}$, and follow the common practice of incorporating it instead in the definition of orbital integrals.

\section{ISOMORPHISMS OF ENDOSCOPIC DATA}

We start by reviewing isomorphism of endoscopic data in the sense of [LS1] and $\mathrm{KS}$. We will then observe the effect of a given isomorphism of endoscopic data on the ingredients of the transfer theorem proved in $\mathbf{S 2}$. The arguments are quite elementary, and we spend most of the section recalling basic definitions and results in order to motivate Lemma 2.4 and Remark 2.5.

We gather definitions from [LS1] and [KS], and follow the notation of [S1, [S2]. Thus $G$ is a connected reductive algebraic group defined over $\mathbb{R}$, and $G^{*}$ is a quasisplit inner form of $G$. We choose a pair $(\psi, u)$, where $\psi: G \rightarrow G^{*}$ is an inner twist and $u$ is a 1-cochain of the Galois group $\Gamma=\{1, \sigma\}$ of $\mathbb{C} / \mathbb{R}$ in $G_{s c}^{*}$ such that $\psi \tau(\psi)^{-1}=$ Int $u(\tau), \tau \in \Gamma$. We write $G^{\vee}$ for the complex dual of $G$, and ${ }^{L} G$ for the $L$-group $G^{\vee} \rtimes W_{\mathbb{R}}$, where the Weil group $W_{\mathbb{R}}$ of $\mathbb{C} / \mathbb{R}$ acts through $W_{\mathbb{R}} \rightarrow \Gamma$.

We recall once again that a set of endoscopic data for $G$ is a tuple $(H, \mathcal{H}, s, \xi)$, where:

(i) $H$ is connected, reductive and quasi-split over $\mathbb{R}$, and so has dual $H^{\vee}$ with dual Galois automorphism $\sigma_{H^{\vee}}$,

(ii) $\mathcal{H}$ is a split extension of $W_{\mathbb{R}}$ by $H^{\vee}$, where $W_{\mathbb{R}}$ acts through $W_{\mathbb{R}} \rightarrow \Gamma$, and now $\sigma$ acts as $\sigma_{H^{\vee}}$ only up to an inner automorphism of $H^{\vee}$,

(iii) $s$ is a semisimple element of $G^{\vee}$, and 
(iv) $\xi: \mathcal{H} \rightarrow{ }^{L} G$ is an embedding of extensions under which the image of $H^{\vee}$ is the identity component of $\operatorname{Cent}\left(s, G^{\vee}\right)$, and the full image lies in Cent $\left(s^{\prime},{ }^{L} G\right)$, for some $s^{\prime}$ congruent to s modulo the center $Z\left(G^{\vee}\right)$ of $G^{\vee}$.

In addition, a $z$-pair for $(H, \mathcal{H}, s, \xi)$ consists of a pair $\left(H_{1}, \xi_{1}\right)$, where $H_{1}$ is a $z$-extension of $H$ and $\xi_{1}$ is an embedding of $\mathcal{H}$ in ${ }^{L} H_{1}$ (Section 2.2 of [KS]). We fix a $z$-pair and write $\lambda_{1}$ for the attached quasicharacter on $Z_{1}(\mathbb{R})$, where $Z_{1}$ is the kernel of $H_{1} \rightarrow H$. For convenience, we require $\lambda_{1}$ to be unitary. We are interested in tempered (irreducible admissible) representations of $H_{1}(\mathbb{R})$ which transform under $Z_{1}(\mathbb{R})$ according to $\lambda_{1}$. Our data provide a map $\Phi_{t e m p}\left(H_{1}, \lambda_{1}\right) \rightarrow \Phi_{t e m p}\left(G^{*}\right)$ on Langlands parameters (see Section 2 of [S2]).

Let $\mathfrak{e}=(H, \mathcal{H}, s, \xi)$ and $\mathfrak{e}^{\prime}=\left(H^{\prime}, \mathcal{H}^{\prime}, s^{\prime}, \xi^{\prime}\right)$ be sets of endoscopic data for $G$. We use $\xi, \xi^{\prime}$ to identify $\mathcal{H}, \mathcal{H}^{\prime}$ as subgroups of ${ }^{L} G$. Combining the discussion of Section 2.1 of [KS] with Section 1.2 of [LS1], we define an isomorphism from $\mathfrak{e}$ to $\mathfrak{e}^{\prime}$ to be an element $g$ of $G^{\vee}$ such that

$$
g \mathcal{H}^{-1}=\mathcal{H}^{\prime}
$$

and

$$
g s g^{-1} \in s^{\prime} Z\left(G^{\vee}\right)\left(Z\left(H^{\prime \vee}\right)^{\Gamma}\right)^{0} .
$$

When this isomorphism $g$ lies in $H^{\vee}$, it is easy to see that there is no effect on the various constructions. In general, we will be concerned only with $g$ modulo right multiplication by an element of $H^{\vee}$, and we write $\beta$ for the restriction of Int $g$ to $H^{\vee}$.

It is convenient at this point to specify some splittings: an $\mathbb{R}$-splitting for each of the various quasi-split groups over $\mathbb{R}$, and a $\Gamma$-invariant splitting for each of the dual groups. Ultimately these choices will have no effect on our results. After multiplication on the right by an element of $H^{\vee}$ we may assume that, for an isomorphism $g$ as above, the attached isomorphism $\beta: H^{\vee} \rightarrow H^{\prime \vee}$ maps $s p l_{H^{\vee}}$ to $s p l_{H^{\prime}}$. Then $\sigma_{H^{\prime} \vee} \circ \beta \circ \sigma_{H^{\vee}}=\beta$. Let $\alpha: H \rightarrow H^{\prime}$ be the isomorphism dual to $\beta^{-1}$ and mapping $s p l_{H}$ to $s p l_{H^{\prime}}$. Then $\alpha$ is defined over $\mathbb{R}$.

We need to extend $\alpha$ to an isomorphism $\alpha_{1}$ of $z$-pairs defined over $\mathbb{R}$ and then we set $\lambda_{1}^{\prime}=\lambda_{1} \circ \alpha_{1}^{-1}$. It is a routine exercise to construct $\left(H_{1}^{\prime}, \xi_{1}^{\prime}\right)$ and then $\alpha_{1}$, and we forgo the details. We will also assume that $s p l_{H_{1}}$ projects to $s p l_{H}$ and $s p l_{H^{\vee}}$ extends to $s p l_{H_{1}^{\vee}}$ in the obvious sense, and similarly for the splittings attached to $H^{\prime}$. We have that $\alpha_{1}$ maps $H_{1}(\mathbb{R})$ isomorphically to $H_{1}^{\prime}(\mathbb{R})$ and $\beta_{1}$ induces a map $\Phi_{\text {temp }}\left(H_{1}, \lambda_{1}\right) \rightarrow \Phi_{\text {temp }}\left(H_{1}^{\prime}, \lambda_{1}^{\prime}\right)$ which respects the map $\pi_{1} \rightarrow \pi_{1}^{\prime}=\pi_{1} \circ \alpha_{1}^{-1}$. Also we write $\gamma_{1}^{\prime}$ for the image $\alpha_{1}\left(\gamma_{1}\right)$ of $\gamma_{1} \in H_{1}(\mathbb{R})$.

We write $\Delta_{\text {spec }}$ and $\Delta_{\text {comp }}$ for the relative spectral and compatibility factors attached (in [S2]) to $(H, \mathcal{H}, s, \xi),\left(H_{1}, \xi_{1}\right)$ and $(\psi, u)$, and $\Delta_{\text {spec }}^{\prime}$ and $\Delta_{\text {comp }}^{\prime}$ for those attached to $\left(H^{\prime}, \mathcal{H}^{\prime}, s^{\prime}, \xi^{\prime}\right),\left(H_{1}^{\prime}, \xi_{1}^{\prime}\right)$ and the same $(\psi, u)$.

\section{Lemma 2.1.}

(i) Let $\gamma_{1} \in H_{1}(\mathbb{R}), \delta \in G(\mathbb{R})$. Then $\left(\gamma_{1}, \delta\right)$ is a strongly $G$-regular related pair relative to $H_{1}$ if and only if $\left(\gamma_{1}^{\prime}, \delta\right)$ is a strongly-regular related pair relative to $H_{1}^{\prime}$.

(ii) Let $\pi_{1}$ be a tempered irreducible representation of $H_{1}(\mathbb{R})$ transforming un$\operatorname{der} Z_{1}(\mathbb{R})$ according to $\lambda_{1}$ and let $\pi$ be a tempered irreducible representation of $G(\mathbb{R})$. Then $\left(\pi_{1}, \pi\right)$ is a $G$-regular related pair for $H_{1}$ if and only if $\left(\pi_{1}^{\prime}, \pi\right)$ is a $G$-regular related pair for $H_{1}^{\prime}$. 
(iii) Let $\left(\pi_{1}, \pi\right),\left(\bar{\pi}_{1}, \bar{\pi}\right)$ be $G$-regular related pairs for $H_{1}$. Then

$$
\Delta_{\text {spec }}\left(\pi_{1}, \pi ; \bar{\pi}_{1}, \bar{\pi}\right)=\Delta_{\text {spec }}^{\prime}\left(\pi_{1}^{\prime}, \pi ; \bar{\pi}_{1}^{\prime}, \bar{\pi}\right) \text {. }
$$

(iv) Let $\left(\pi_{1}, \pi\right)$ be a $G$-regular related pair and $\left(\gamma_{1}, \delta\right)$ be a strongly $G$-regular related pair for $H_{1}$. Then

$$
\Delta_{\text {comp }}\left(\pi_{1}, \pi ; \gamma_{1}, \delta\right)=\Delta_{c o m p}^{\prime}\left(\pi_{1}^{\prime}, \pi ; \gamma_{1}^{\prime}, \delta\right) .
$$

Proof. At this point we pause to review the definition of admissibility (and of toral data). First, $\eta_{1}$ is a composition of the projection $T_{1} \rightarrow T_{1} / Z_{1}=T_{H}$ with an admissible embedding $\eta: T_{H} \rightarrow T$. This means that $\eta$ is defined over $\mathbb{R}$ and is the composition of an admissible embedding $\eta^{*}: T_{H} \rightarrow T^{*}$ of the maximal torus $T_{H}$ in the quasi-split form $G^{*}$ and the inverse of a modified twist Int $x \circ \psi: T \rightarrow T^{*}$ over $\mathbb{R}$. Thus it is the isomorphism $\eta^{*}$ that we need to describe. We have chosen splittings of $H, H^{\vee}, G^{*}$ and $G^{\vee}$, and may then identify the chosen maximal torus $\mathcal{T}_{H}$ in $H^{\vee}$ with that, $\mathcal{T}$, in $G^{\vee}$. The choice of Borel subgroups in $H$ and $G^{*}$ containing, respectively, $T_{H}$ and $T^{*}$ determines isomorphisms $T_{H}^{\vee} \rightarrow \mathcal{T}_{H}$ and $\mathcal{T} \rightarrow T^{* \vee}$, and then by duality $\eta^{*}: T_{H} \rightarrow T^{*}$. We call $\eta^{*}$ admissible when the Borel subgroups are chosen so that $\eta^{*}$ is defined over $\mathbb{R}$ (see Section 1.3 of [LS1]), and also write $\eta_{1}^{*}$ for the composition of $\eta^{*}$ with the projection $T_{1} \rightarrow T_{1} / Z_{1}=T_{H}$. For convenience, we call $\eta_{1}^{*}$ quasi-split toral data and $\eta_{1}$ toral data for $G$, leaving implicit the role of the Borel subgroups.

We claim that $\eta_{1}: T_{1} \rightarrow T$ is admissible for $H$ if and only if $\eta_{1} \circ \alpha_{1}^{-1}: T_{1}^{\prime} \rightarrow T$ is admissible for $H^{\prime}$. Since $\alpha$ is defined by Int $g: \mathcal{T} \rightarrow \mathcal{T}$ and both $\eta_{1}$ and $\eta_{1} \circ \alpha_{1}^{-1}$ are defined over $\mathbb{R}$, this, as well as (i), is clear from the definitions. Also (ii) reduces to a well-known property of the Langlands parametrization. For (iii) and (iv) we observe, by inspecting each construction, that the same assertions are true for the individual terms $\Delta_{I}, \Delta_{I I}$ and $\Delta_{I I I}$. Here we choose toral data as in the argument for (i) and use the same $a$-data for $T$ in each case, transporting $a$-data for $H$ and $H^{\prime}$ accordingly.

Corollary 2.2. Let $\Delta$ be a spectral transfer factor for $H$ and define a function $\Delta^{\prime}$ for $H^{\prime}$ by

$$
\Delta^{\prime}\left(\pi_{1}^{\prime}, \pi\right)=\Delta\left(\pi_{1}^{\prime} \circ \alpha_{1}, \pi\right) .
$$

Then $\Delta^{\prime}$ is a spectral transfer factor for $H^{\prime}$.

Proof. We have to show that

$$
\Delta^{\prime}\left(\pi_{1}^{\prime}, \pi\right) / \Delta^{\prime}\left(\bar{\pi}_{1}^{\prime}, \bar{\pi}\right)=\Delta_{\text {spec }}^{\prime}\left(\pi_{1}^{\prime}, \pi ; \bar{\pi}_{1}^{\prime}, \bar{\pi}\right) .
$$

But

$$
\begin{aligned}
& \Delta^{\prime}\left(\pi_{1}^{\prime}, \pi\right) / \Delta^{\prime}\left(\bar{\pi}_{1}^{\prime}, \bar{\pi}\right) \\
& \quad=\Delta\left(\pi_{1}^{\prime} \circ \alpha_{1}, \pi\right) / \Delta\left(\bar{\pi}_{1}^{\prime} \circ \alpha_{1}, \bar{\pi}\right) \\
& \quad=\Delta_{\text {spec }}\left(\pi_{1}^{\prime} \circ \alpha_{1}, \pi ; \bar{\pi}_{1}^{\prime} \circ \alpha_{1}, \bar{\pi}\right) .
\end{aligned}
$$

So the result follows from (iii).

Now write these absolute factors as $\Delta_{\text {spec }}, \Delta_{\text {spec }}^{\prime}$. Arthur discusses the analogous geometric factors $\Delta_{\text {geom }}, \Delta_{\text {geom }}^{\prime}$ in [A1] (or we could argue directly as in Lemma 2.1 and Corollary 2.2).

Corollary 2.3. $\Delta_{\text {spec }}^{\prime}$ and $\Delta_{\text {geom }}^{\prime}$ are compatible if and only if the same is true for $\Delta_{\text {spec }}$ and $\Delta_{\text {geom }}$. 
Proof. This is immediate from (iv).

We now apply this to the transfer theorem ([S2], Theorem 5.1). Thus we fix an isomorphism $(H, \mathcal{H}, s, \xi) \rightarrow\left(H^{\prime}, \mathcal{H}^{\prime}, s^{\prime}, \xi^{\prime}\right)$, attach $z$-pair $\left(H_{1}^{\prime}, \xi_{1}^{\prime}\right)$ to given $\left(H_{1}, \xi_{1}\right)$ as above, and also attach compatible factors $\Delta_{\text {geom }}^{\prime}, \Delta_{\text {spec }}^{\prime}$ to given compatible $\Delta_{\text {geom }}, \Delta_{\text {spec }}$ as in Corollaries 2.2 and 2.3. Then the following is immediate, first for the $G$-regular case, and then in general by applying the coherent continuation of Section 14 of $\underline{\mathrm{S} 2}$.

Lemma 2.4. Suppose in the hypothesis of the transfer theorem we replace $(H, \mathcal{H}, s, \xi)$ by $\left(H^{\prime}, \mathcal{H}^{\prime}, s^{\prime}, \xi^{\prime}\right),\left(H_{1}, \xi_{1}\right)$ by $\left(H_{1}^{\prime}, \xi_{1}^{\prime}\right)$ and $\Delta_{\text {geom }}, \Delta_{\text {spec }}$ by $\Delta_{\text {geom }}^{\prime}$, $\Delta_{\text {spec }}^{\prime}$. Then in the conclusion of the theorem we may replace $f_{1}$ by $f_{1}^{\prime}=f_{1} \circ \alpha_{1}^{-1}$. Moreover, whatever the choice we make for $f_{1}^{\prime}$, we have

$$
\text { St-Tr } \pi_{1}\left(f_{1}\right)=S t-\operatorname{Tr} \pi_{1}^{\prime}\left(f_{1}^{\prime}\right)
$$

for all tempered irreducible $\pi_{1}$.

Remark 2.5. If $g$ is an isomorphism from $\mathfrak{e}=(H, \mathcal{H}, s, \xi)$ to $\mathfrak{e}^{\prime}=\left(H^{\prime}, \mathcal{H}^{\prime}, s^{\prime}, \xi^{\prime}\right)$, we set $\mathfrak{e}^{\prime}=g \cdot \mathfrak{e}$ and $\Delta^{\prime}=g . \Delta$. As Arthur explains in A1, if $G$ is quasi-split, then we can extend a normalization for the absolute transfer factors $\Delta$ for given $\mathfrak{e}$ to its isomorphism class by attaching $g . \Delta$ to $g . \mathfrak{e}$, but that, for a general reductive group $G$, $g$ may fix $\mathfrak{e}$ yet act nontrivially on $\Delta$. As we have just seen, this does not concern us in the statement of the transfer theorem, nor will it matter in the inversion identity where the terms are independent of the normalization of $\Delta$ (see Section 7 ). It does concern us for the normalization of endoscopic characters (see Section 3), and again when we come to the structure of $L$-packets in Section 11. Arthur's analysis of $g . \Delta$ in A1 motivates his statement of the property we prove in Corollary 11.2. This then yields intrinsic normalizations for all endoscopic characters (see Lemma 11.4).

\section{TEMPERED ENDOSCOPIC CHARACTERS}

We will call the distribution

$$
f \rightarrow S t-\operatorname{Tr} \pi_{1}\left(f_{1}\right),
$$

which we have shown in the transfer theorem $[\mathbf{S 2}$ to coincide with the tempered virtual character

$$
f \rightarrow \sum_{\pi} \Delta_{\text {spec }}\left(\pi_{1}, \pi\right) \operatorname{Tr} \pi(f),
$$

an endoscopic character on $G(\mathbb{R})$. It evidently depends on the choice of normalization for the compatible factors $\Delta_{\text {geom }}, \Delta_{\text {spec }}$. Such factors may only be multiplied by a common constant, and all endoscopic characters attached to a given set of endoscopic data and given $z$-pair are then multiplied by the same constant. In the inversion formula we will multiply each endoscopic character by a coefficient with cancelling dependence; see Lemma 7.1.

We have seen in Lemma 2.4 that endoscopic characters are unchanged when the endoscopic data is modified by an isomorphism, provided we use the attached modification of transfer factors. Next we note that the choice of $z$-pair works similarly. Suppose that $\left(H_{1}, \xi_{1}\right)$ and $\left(H_{2}, \xi_{2}\right)$ are both $z$-pairs for $(H, \mathcal{H}, s, \xi)$. Then we have a bijection $\Phi_{\text {temp }}\left(H_{1}, \lambda_{1}\right) \rightarrow \Phi_{\text {temp }}\left(H_{2}, \lambda_{2}\right)$, which we will write as $\varphi_{1} \rightarrow$ $\varphi_{2}$, obtained by choosing a representative $\varphi_{1}$ for $\varphi_{1}$ with image in $\xi_{1}(\mathcal{H})$ and mapping it to the class $\varphi_{2}$ of $\varphi_{2}=\xi_{2} \circ \xi_{1}^{-1} \circ \varphi_{1}$. Let $\pi_{1}, \pi_{2}$ have parameters $\varphi_{1}, \varphi_{2}$ 
respectively, and let $\pi$ be a tempered irreducible representation of $G(\mathbb{R})$. Then $\left(\pi_{1}, \pi\right)$ is a $(G$-regular $)$ related pair for $\left(H_{1}, \xi_{1}\right)$ if and only if $\left(\pi_{2}, \pi\right)$ has the same property for $\left(H_{2}, \xi_{2}\right)$.

Lemma 3.1. We may normalize the spectral transfer factors for $\left(H_{1}, \xi_{1}\right)$ and $\left(H_{2}, \xi_{2}\right)$ so that

$$
\Delta_{\text {spec }}\left(\pi_{2}, \pi\right)=\Delta_{\text {spec }}\left(\pi_{1}, \pi\right) .
$$

Proof. We start with the $G$-regular case. As in the last section we just have to show that the relative factors coincide, i.e.,

$$
\Delta_{\text {spec }}\left(\pi_{2}, \pi ; \bar{\pi}_{2}, \bar{\pi}\right)=\Delta_{\text {spec }}\left(\pi_{1}, \pi ; \bar{\pi}_{1}, \bar{\pi}\right) .
$$

A check of the definitions shows that this is true when $\Delta_{\text {spec }}$ is replaced by each of $\Delta_{I}, \Delta_{I I}, \Delta_{I I I}$ using the corresponding toral data and same $a$-data, and so we are done in the $G$-regular case. For the general case, we recall again the definition of $\Delta_{\text {spec }}\left(\pi_{i}, \pi\right)$ by coherent continuation, and the lemma follows.

If we replace $\left(H_{1}, \xi_{1}\right)$ by $\left(H_{2}, \xi_{2}\right)$ we will use Lemma 3.1 to define the new spectral transfer factor, and then apply the canonical compatibility factors to fix the normalization of the geometric factors as in Section 4 of [ $\underline{\mathrm{S} 2}$, and so avoid a direct discussion of the geometric factors which would take a little longer (see Remark 11.3 of [S1]).

Lemma 3.2. Suppose in the hypothesis of the transfer theorem that we replace the $z$-pair $\left(H_{1}, \xi_{1}\right)$ by another $z$-pair $\left(H_{2}, \xi_{2}\right)$, with the modification of transfer factors described above. Then if $f_{1}$ is replaced by $f_{2}$ in the conclusion, we have

$$
\operatorname{St}-\operatorname{Tr} \pi_{2}\left(f_{2}\right)=\operatorname{St}-\operatorname{Tr} \pi_{1}\left(f_{1}\right) \text {. }
$$

Proof. We have arranged the normalization of spectral transfer factors so that the formulas for $S t-\operatorname{Tr} \pi_{1}\left(f_{1}\right)$ and $S t-T r \pi_{2}\left(f_{2}\right)$ given by the transfer theorem are identical.

We emphasize that, according to our definition, stable tempered characters on $G(\mathbb{R})$ are endoscopic. Notice that if we use the trivial endoscopic data $\left(G^{*},{ }^{L} G, 1, i d\right)$ and normalize transfer so that the geometric transfer factors are either one or zero, then the associated endoscopic characters are the usual stable characters attached to tempered $L$-packets, but now multiplied by the constant $(-1)^{q_{G}-q_{G^{*}}}$. We may of course arrange to insert this constant in the definition of the geometric transfer factors instead, if we wish. Whichever way is chosen, $(-1)^{q_{G}-q_{G^{*}}}$ appears in the formulas when we write the spectral transfer as a set of identities for the characters as analytic functions on the regular semisimple set of $G(\mathbb{R})$.

\section{REview of $K$-Groups And EXTENDED GEOMETRIC FACTORS}

We then come to the role of the twisting data $(\psi, u)$ in the transfer theorem. First of all, notice that, for a given twist $\psi$, the choice of cochain $u$ has no effect on the relative transfer factors: $u$ potentially affects only $\Delta_{I I I_{1}}$ and there the choice does not matter (see p. 31 of [LS1]). The argument for relative spectral factors is the same. Moreover, it is only the inner class of $\psi$ that matters, i.e. the factors are unchanged if we replace $\psi$ by $\psi^{\prime}=$ Int $g \circ \psi$, with $g \in G^{*}$. In that case we may replace $u$ by $u^{\prime}$ given by $u^{\prime}(\tau)=g_{s c} u(\tau) \tau\left(g_{s c}\right)^{-1}, \tau \in \Gamma$, where $g_{s c} \in G_{s c}^{*}$ and $g$ have the same image in $G_{a d}^{*}$. 
We prepare now to work simultaneously with several pairs $\left(G^{\prime}, \psi^{\prime}\right)$. We follow Arthur's discussion in [A2] of $K$-groups and extended geometric factors based on constructions due to Kottwitz.

To start, we recall certain inner forms of $G$. Throughout, $(G, \psi, u)$ is fixed. Consider also $\left(G^{\prime}, \psi^{\prime}, u^{\prime}\right)$ and write $\psi^{\prime}$ as $\psi \circ \xi$, and $\xi \circ \tau(\xi)^{-1}$ as Int $x_{s c}(\tau)$, where $x_{s c}(\tau)$ lies in $G_{s c}, \tau \in \Gamma$. We are interested in groups $G^{\prime}$ for which $x_{s c}$ is a 1-cocycle of $\Gamma$ in $G_{s c}$. We may assume that $u^{\prime}=\psi_{s c}\left(x_{s c}\right) u$. Since $\partial u^{\prime}$ is easily calculated to be $\psi_{s c}\left(\partial x_{s c}\right) \partial u$, we obtain $\partial u^{\prime}=\partial u$. Conversely, if given instead that $\partial u^{\prime}=\partial u$ we see that $x_{s c}=\psi_{s c}^{-1}\left(u^{\prime} u^{-1}\right)$ is a 1 -cocycle of $\Gamma$ in $G_{s c}$. We will make use of the following.

\section{Lemma 4.1.}

$$
(-1)^{q_{G}-q_{G^{*}}}=(-1)^{q_{G^{\prime}}-q_{G^{*}}},
$$

where $2 q_{G}$ is the dimension of the symmetric space attached to $G_{s c}$.

Proof. This is immediate from $\mathrm{K} 2$. On pp. $295-296$ it is proved that $(-1)^{q_{G}-q_{G^{*}}}$ is the Kottwitz sign $e(G)$ for a group $G$ over $\mathbb{R}$, and in the definition of $e(G)$ on pp. 291-292, we have that $u_{a d}$ and $u_{a d}^{\prime}$ map to the class of $\partial u=\partial u^{\prime}$ under

$$
H^{1}\left(\Gamma, G_{a d}\right) \rightarrow H^{2}\left(\Gamma, Z\left(G_{s c}\right)\right) .
$$

The rest of the definition is the same for $G$ and $G^{\prime}$, so that $e(G)=e\left(G^{\prime}\right)$.

The prescription of $\mathrm{A} 2$ is to consider one inner form $G^{\prime}$ for each element of the image of the map $H^{1}\left(\Gamma, G_{s c}\right) \rightarrow H^{1}(\Gamma, G)$. Some small examples are useful to keep in mind. If $G$ is $S L(2)$, then we get nothing new, whereas if $G$ is $S U(2)$, then we have two cohomology classes, and each of these yield the compact form $S U(2)$ as $G^{\prime}$. So for $G=S L(2)$ we will work with one copy of $S L(2)$, but for $G=S U(2)$ we will work with two copies of $S U(2)$. On the other hand, the case $G=S U(3)$ is nicer: we work with one copy of each of $S U(3)$ and $S U(2,1)$. Starting instead with $S U(2,1)$ leads to the same result.

These examples also illustrate very simply what we gain from the $K$-group construction. If we consider $G=G_{s c}=S U(2,1)$, then a stable conjugacy class of regular elliptic elements consists of three conjugacy classes parametrized by three of the four elements of $H^{1}(\Gamma, T)$, where $T$ is a compact maximal torus, and similarly an $L$-packet of discrete series representations is parametrized by three elements of the same group. The $K$-group construction finds for us a fourth conjugacy class and a fourth representation from $S U(3)$. Similarly, if we start with $S U(3)$, then we add three classes or representations from $S U(2,1)$. On the other hand, in the case of $G=G_{s c}=S L(2)=S U(1,1)$ no additions are needed: conjugacy classes in a stable conjugacy class of regular elliptic elements and $L$-packets of discrete series representations are each parametrized by the two element group $H^{1}(\Gamma, T)$. When we come to $S U(2)$, however, we are missing a conjugacy class and a representation. The second copy of $S U(2)$ provides these. In terms of Langlands' original definitions [L1], the construction expands the parametrization set

$$
\mathcal{D}(T)=\operatorname{Ker}\left(H^{1}(\Gamma, T) \rightarrow H^{1}(\Gamma, G)\right),
$$

which is a subset of the group

$$
\mathcal{E}(T)=\operatorname{Im}\left(H^{1}\left(\Gamma, T_{s c}\right) \rightarrow H^{1}(\Gamma, T)\right),
$$

to all of $\mathcal{E}(T)$. The group $\mathcal{E}(T)$ is the same in each inner form $G^{\prime}$ sharing $T$, whereas $\mathcal{D}(T)$ is not. The $K$-group construction allows us to identify $\mathcal{E}(T)$ with the disjoint 
union of the contributing $\mathcal{D}(T)$. We recall the argument in Section 8 (see the proof of Theorem 7.5).

In general, we choose a set of 1-cocycles $x_{s c}^{j}$ of $\Gamma$ in $G_{s c}$ (and identify $x_{s c}^{j}$ with $x_{s c}^{j}(\sigma)$ when convenient) for which the images $x^{j}$ in $G$ represent the classes in the image of the map $H^{1}\left(\Gamma, G_{s c}\right) \rightarrow H^{1}(\Gamma, G), j=1,2, \ldots$, and then attach a set of triples $\left(G^{j}, \psi^{j}, u^{j}\right)$, with $\left(G^{1}, \psi^{1}, u^{1}\right)=(G, \psi, u)$ and $u^{j}=\psi_{s c}\left(x_{s c}^{j}\right) u$. That is enough to specify a frame for a $K$-group as in Section 1.2 of $\mathrm{A} 2$. This $K$-group is an algebraic variety defined over $\mathbb{R}$ which we denote by $\mathbf{G}$. We identify $\mathbf{G}(\mathbb{C}), \mathbf{G}(\mathbb{R})$ with the disjoint union of the groups $G^{j}(\mathbb{C}), G^{j}(\mathbb{R})$ respectively, writing $\mathbf{G}=\bigsqcup_{j} G^{j}$, etc. Also, we write $\psi^{j k}$ for the inner twist $\left(\psi^{k}\right)^{-1} \circ \psi^{j}$ from $G^{j}$ to $G^{k}$, and then $\left(\psi^{1 k}\right)^{-1} \sigma\left(\psi^{1 k}\right)=$ Int $x_{s c}^{k}$.

The image $x^{j}$ in $G$ of $x_{s c}^{j}$ is also a cocycle. Its class in $H^{1}(\Gamma, G)$ determines a character on $A(G)=\pi_{0}\left(Z\left(G^{\vee}\right)^{\Gamma}\right)$ K1. This character is, by design, the trivial character [K1. At the same time, for all $j$, the image $u_{a d}^{j}$ of $u^{j}$ in $G_{a d}^{*}$ determines the same character $\zeta_{\mathbf{G}}$ on $A\left(G_{a d}^{*}\right)=A\left(G_{a d}\right)=\left(Z_{s c}^{\vee}\right)^{\Gamma}$, where $Z_{s c}^{\vee}$ is the center of the simply-connected cover $G_{s c}^{\vee}$ of the adjoint group for $G^{\vee}$ (see, particularly, 1.4 of [K1]). We will mention this character $\zeta_{\mathbf{G}}$ explicitly in Section 11, and in the proof of Theorem 7.5 for the singular elliptic case (Section 9). More specifically, in that proof we use the character $\zeta_{\mathbf{M}}^{\mathbf{G}}$ on $\pi_{0}\left(Z\left(M_{s c}^{\vee}\right)^{\Gamma}\right)$, attached to $\zeta_{\mathbf{G}}$ by Arthur in Section 2 of [A2, to interpret results we would otherwise deduce directly from [S3] in a less appealing form. Here $\mathbf{M}$ denotes a cuspidal Levi group in $\mathbf{G}$ (see [A2] for the fundamental results) and, as is customary, $M_{s c}^{\vee}$ denotes the inverse image of the adjoint group of $M^{\vee}$ in the simply-connected form attached to $G^{\vee}$ rather than in that attached to $M^{\vee}$.

A stable conjugacy class in $\mathbf{G}(\mathbb{R})$ is the union of matching stable conjugacy classes in the inner forms $G^{j}(\mathbb{R})$. In particular, strongly regular elements $\delta^{j}$ and $\delta^{k}$, in $G^{j}(\mathbb{R})$ and $G^{k}(\mathbb{R})$, respectively, are stably conjugate if and only if $\psi^{j k}\left(\delta^{j}\right)$ is conjugate to $\delta^{k}$ in $G^{k}(\mathbb{C})$. For general regular semisimple $\delta^{j}$ and $\delta^{k}$ we add the usual requirement that the conjugation be given by an element $g^{k}$ of $G^{k}(\mathbb{C})$ such that the restriction of Int $g^{k} \circ \psi^{j k}$ to the identity component of $\operatorname{Cent}\left(\delta^{j}, G^{j}\right)$ is defined over $\mathbb{R}$. If the maximal torus $T_{\delta^{j}}=\operatorname{Cent}\left(\delta^{j}, G^{j}\right)^{0}$ is fundamental (maximally compact) in $G^{j}$, then there is a matching conjugacy class in $G^{k}(\mathbb{R})$ for every $k$ ([S4, Lemma 2.8).

We turn now to endoscopy and extended transfer factors. Relative factors concern us first. Fix a set $(H, \mathcal{H}, s, \xi)$ of endoscopic data and a $z$-pair $\left(H_{1}, \xi_{1}\right)$. We recall the geometric factor for $\mathbf{G}$ which we will denote $\boldsymbol{\Delta}_{\text {geom }}$.

We follow Kottwitz's definition A2 to specify $\boldsymbol{\Delta}_{\text {geom }}\left(\gamma_{1}, \boldsymbol{\delta} ; \gamma_{1}^{\prime}, \boldsymbol{\delta}^{\prime}\right)$, for strongly G-regular related pairs $\left(\gamma_{1}, \boldsymbol{\delta}\right),\left(\gamma_{1}^{\prime}, \boldsymbol{\delta}^{\prime}\right)$ in $H_{1}(\mathbb{R}) \times \mathbf{G}(\mathbb{R})$. This means, of course, that $\boldsymbol{\delta}=\delta^{j} \in G^{j}(\mathbb{R})$ and $\boldsymbol{\delta}^{\prime}=\delta^{k} \in G^{k}(\mathbb{R})$ for some $j, k$, and that $\left(\gamma_{1}, \delta^{j}\right),\left(\gamma_{1}^{\prime}, \delta^{k}\right)$ are strongly $G^{j}-, G^{k}$-regular related pairs in $H_{1}(\mathbb{R}) \times G^{j}(\mathbb{R})$ and $H_{1}(\mathbb{R}) \times G^{k}(\mathbb{R})$, respectively. If $j=k$ we revert to our old notation of $\delta, \delta^{\prime}$ instead of $\delta^{j}, \delta^{k}$. The factor $\boldsymbol{\Delta}_{\text {geom }}$ is the product of three terms which we write as $\boldsymbol{\Delta}_{I}, \boldsymbol{\Delta}_{I I+}, \boldsymbol{\Delta}_{I I I_{1}}$. See [S1 for our grouping, and recall that we now remove the original $\Delta_{I V}$ from $\Delta_{I I+}$. We fix toral data for each of the pairs $\left(\gamma_{1}, \delta^{j}\right),\left(\gamma_{1}^{\prime}, \delta^{k}\right)$ relative to $\mathbf{G}$, by which we mean that we use the same quasi-split toral data whatever the index $j$ or $k$, and then extend this homomorphism to $G^{j}, G^{k}$ by means of the chosen twists $\psi^{j}, \psi^{k}$ (see the proof of Lemma 2.1 to recall definitions). These data then allow us to 
generate $a$-data in each $G^{j}$ from a single set of $a$-data in the quasi-split form, if we wish.

Each of $\Delta_{I}, \Delta_{I I+}$ is a quotient of absolute terms, so there is nothing new to do:

$$
\boldsymbol{\Delta}_{I}\left(\gamma_{1}, \boldsymbol{\delta} ; \gamma_{1}^{\prime}, \boldsymbol{\delta}^{\prime}\right)=\boldsymbol{\Delta}_{I}\left(\gamma_{1}, \boldsymbol{\delta}\right) / \boldsymbol{\Delta}_{I}\left(\gamma_{1}^{\prime}, \boldsymbol{\delta}^{\prime}\right)=\Delta_{I}\left(\gamma_{1}, \delta^{j}\right) / \Delta_{I}\left(\gamma_{1}^{\prime}, \delta^{k}\right)
$$

and so on. The definition of $\boldsymbol{\Delta}_{I I I_{1}}$ requires more care. Following A2, we return to Section 3.4 of [LS1, and use $\delta^{j}$ to make the cochain $v^{j}$ of $\Gamma$ in the maximal torus $T_{s c}^{*}$ in $G_{s c}^{*}$ provided by the chosen toral data, and similarly we use $\delta^{k}$ in $T_{s c}^{* \prime}$ to make $v^{k}$. Set $U=U\left(T_{s c}^{*}, T_{s c}^{* \prime}\right)$ in the notation of [LS1]. Then since

$$
\partial v^{j}=\partial u^{j}=\partial u^{k}=\partial v^{k}
$$

we obtain a well-defined cocycle in $U$ from $\left(\left(v^{j}\right)^{-1}, v^{k}\right)$, and the rest of the construction of $\boldsymbol{\Delta}_{I I I_{1}}\left(\gamma_{1}, \boldsymbol{\delta} ; \gamma_{1}^{\prime}, \boldsymbol{\delta}^{\prime}\right)$ proceeds as in [LS1]. Because $\boldsymbol{\Delta}_{I}, \boldsymbol{\Delta}_{I I+}$ depend only on the quasi-split form $G^{*}$ rather than on $G^{j}$ and $G^{k}$, the argument that $\boldsymbol{\Delta}_{\text {geom }}\left(\gamma_{1}, \boldsymbol{\delta} ; \gamma_{1}^{\prime}, \boldsymbol{\delta}^{\prime}\right)$ is canonical also proceeds as in [LS1]. by

The absolute geometric transfer factor $\boldsymbol{\Delta}\left(\gamma_{1}, \boldsymbol{\delta}\right)=\boldsymbol{\Delta}_{\text {geom }}\left(\gamma_{1}, \boldsymbol{\delta}\right)$ is then defined

$$
\boldsymbol{\Delta}\left(\gamma_{1}, \boldsymbol{\delta}\right)=\boldsymbol{\Delta}_{g e o m}\left(\gamma_{1}, \boldsymbol{\delta} ; \bar{\gamma}_{1}, \overline{\boldsymbol{\delta}}\right) \boldsymbol{\Delta}\left(\bar{\gamma}_{1}, \overline{\boldsymbol{\delta}}\right),
$$

where $\left(\bar{\gamma}_{1}, \overline{\boldsymbol{\delta}}\right)=\left(\bar{\gamma}_{1}, \overline{\delta^{j}}\right)$ is some fixed strongly $G$-regular related pair and $\boldsymbol{\Delta}\left(\bar{\gamma}_{1}, \overline{\boldsymbol{\delta}}\right)$ is chosen arbitrarily. Thus a uniform normalization of the absolute geometric factors $\Delta\left(\gamma_{1}, \delta^{j}\right)$ is achieved for the groups $G^{j}$, strengthening the relative local hypothesis of Corollary 4.2.B of [LS1] for general inner forms.

\section{EXTENDED SPECTRAL FACTORS AND COMPATIBILITY}

On the spectral side, we use the conventions of S2 for Langlands parameters. Thus $\Phi_{t e m p}\left(G^{j}\right)$ consists of the parameters in $\Phi_{t e m p}\left(G^{*}\right)$ that are relevant to $G^{j}$ with respect to $\psi^{j}$. We write such a parameter as $\varphi^{j}$ and typically distinguish it in notation from its image $\varphi^{*}$ under the inclusion of $\Phi_{t e m p}\left(G^{j}\right)$ in $\Phi_{\text {temp }}\left(G^{*}\right)$. Then $\Phi_{\text {temp }}(\mathbf{G})$ consists of the parameters in $\Phi_{t e m p}\left(G^{*}\right)$ that are relevant to at least one $G^{j}$. Any discrete parameter (if such exists) is relevant to all $G^{j}$. We write $\varphi$ for a typical parameter for $\mathbf{G}$, so that $\varphi=\varphi^{j}$ for at least one $j$. The $L$-packet attached to $\varphi \in \Phi_{\text {temp }}(\mathbf{G})$ (or extended $L$-packet for emphasis) is the union of the $L$-packets for those $\varphi^{j}$ for which $\varphi=\varphi^{j}$.

The stable characters attached to tempered $L$-packets are invariant under inner twisting (in the sense of S5]) up to a sign which in the case of the twist $\psi^{j k}$ is $(-1)^{q_{G}}-q_{G^{j}}$. By Lemma 4.1 this sign is trivial. That implies that the characters $\chi_{\boldsymbol{\varphi}^{j}}$ attached to those $L$-packets forming a tempered $\mathbf{L}$-packet for $\mathbf{G}$ determine a stably invariant function $\chi_{\boldsymbol{\varphi}}$ on the regular semisimple set of $\mathbf{G}(\mathbb{R})$ by the simple patching formula

$$
\chi_{\boldsymbol{\varphi}}(\boldsymbol{\delta})=\chi_{\boldsymbol{\varphi}^{j}}\left(\delta^{j}\right)
$$

for $\boldsymbol{\delta}$ stably conjugate to regular semisimple $\delta^{j}$ in $\mathbf{G}(\mathbb{R})$. This function represents the tempered distribution on $\mathbf{G}(\mathbb{R})$ that we will denote $\operatorname{St}-\operatorname{Tr}(\boldsymbol{\pi})$, for any $\boldsymbol{\pi}$ in the L-packet.

By spectral G-regular related pairs $\left(\pi_{1}, \boldsymbol{\pi}\right),\left(\pi_{1}^{\prime}, \boldsymbol{\pi}^{\prime}\right)$ we mean $G^{j}-, G^{k}$-regular related pairs $\left(\pi_{1}, \pi^{j}\right),\left(\pi_{1}^{\prime}, \pi^{k}\right)$, for some $j, k$ in the sense of S2. If $j=k$ we switch back to the notation $\pi, \pi^{\prime}$ instead of $\pi^{j}, \pi^{k}$. Thus $\pi_{1}, \pi_{1}^{\prime}$ have parameters $\boldsymbol{\varphi}_{1}, \boldsymbol{\varphi}_{1}^{\prime} \in \Phi_{\text {temp }}\left(H_{1}, \lambda_{1}\right)$ which have images $\varphi^{*}, \boldsymbol{\varphi}^{* \prime}$ in $\Phi_{t e m p}\left(G^{*}\right)$ and images $\varphi^{j}, \varphi^{k}$ 
in $\Phi_{\text {temp }}\left(G^{j}\right)$ which are the parameters for $\pi^{j}, \pi^{k}$. Our next step is to use the parallel constructions of [S2] to define

$$
\boldsymbol{\Delta}_{\text {spec }}\left(\pi_{1}, \boldsymbol{\pi} ; \pi_{1}^{\prime}, \boldsymbol{\pi}^{\prime}\right)=\boldsymbol{\Delta}\left(\pi_{1}, \pi^{j} ; \pi_{1}^{\prime}, \pi^{k}\right)
$$

as a product $\boldsymbol{\Delta}_{I} \boldsymbol{\Delta}_{I I} \boldsymbol{\Delta}_{I I I}$.

We pick toral data relative to $\mathbf{G}$, as well as $a$-data. Again there is nothing new to do for $\boldsymbol{\Delta}_{I}, \boldsymbol{\Delta}_{I I}$ since they are each a quotient of absolute terms (see Sections 8 and 9 of $\underline{\underline{S} 2]}$ :

$$
\boldsymbol{\Delta}_{I}\left(\pi_{1}, \pi^{j} ; \pi_{1}^{\prime}, \pi^{k}\right)=\Delta_{I}\left(\pi_{1}, \pi^{j}\right) / \Delta_{I}\left(\pi_{1}^{\prime}, \pi^{k}\right),
$$

and

$$
\boldsymbol{\Delta}_{I I}\left(\pi_{1}, \pi^{j} ; \pi_{1}^{\prime}, \pi^{k}\right)=\Delta_{I I}\left(\pi_{1}, \pi^{j}\right) / \Delta_{I I}\left(\pi_{1}^{\prime}, \pi^{k}\right) .
$$

For $\boldsymbol{\Delta}_{I I I}$ we adapt the method for geometric $\boldsymbol{\Delta}_{I I I_{1}}$, now using Section 10 of [S2] to make a cocycle $\left(\left(v^{j}\right)^{-1}, v^{k}\right)$ in the torus $U=U\left(T_{s c}^{*}, T_{s c}^{* \prime}\right)$. It is routine then to show that $\boldsymbol{\Delta}_{\text {spec }}\left(\pi_{1}, \pi^{j} ; \pi_{1}^{\prime}, \pi^{k}\right)$ is canonical. The arguments again come from [LS1] for the effect of choices on $\boldsymbol{\Delta}_{I}, \boldsymbol{\Delta}_{I I I}$. For $\boldsymbol{\Delta}_{I I}$, Lemma 9.3 of [S2] shows that the behavior is correct under change of toral data and change of $a$-data.

We may now fix a normalization of the absolute spectral factor

$$
\boldsymbol{\Delta}_{\text {spec }}\left(\pi_{1}, \boldsymbol{\pi}\right)=\boldsymbol{\Delta}\left(\pi_{1}, \boldsymbol{\pi}\right)
$$

by the standard device. Thus we fix a G-regular related pair $\left(\bar{\pi}_{1}, \overline{\boldsymbol{\pi}}\right)$ and fix $\boldsymbol{\Delta}\left(\bar{\pi}_{1}, \overline{\boldsymbol{\pi}}\right)$ as we wish. Then for all G-regular related pairs $\left(\pi_{1}, \boldsymbol{\pi}\right)$ we define

$$
\boldsymbol{\Delta}\left(\pi_{1}, \boldsymbol{\pi}\right)=\boldsymbol{\Delta}_{\text {spec }}\left(\pi_{1}, \boldsymbol{\pi} ; \bar{\pi}_{1}, \overline{\boldsymbol{\pi}}\right) \boldsymbol{\Delta}\left(\bar{\pi}_{1}, \overline{\boldsymbol{\pi}}\right) .
$$

In particular, we have then a normalization of the absolute spectral factors $\Delta\left(\pi_{1}, \pi^{j}\right)$ for each group $G^{j}$. We extend these factors for each group to all tempered related pairs $\left(\pi_{1}, \pi^{j}\right)$ by coherent continuation as in [S2. This yields a well-defined factor $\boldsymbol{\Delta}\left(\pi_{1}, \boldsymbol{\pi}\right)$ for each tempered related pair in $\mathbf{G}$ (or we could argue by a coherent continuation directly on $\mathbf{G}(\mathbb{R})$ ).

Similarly we may define an extended compatibility factor

$$
\boldsymbol{\Delta}_{\text {comp }}\left(\pi_{1}, \boldsymbol{\pi} ; \gamma_{1}, \boldsymbol{\delta}\right)
$$

and formulate compatibility of the absolute factors $\boldsymbol{\Delta}\left(\gamma_{1}, \boldsymbol{\delta}\right)$ and $\boldsymbol{\Delta}\left(\pi_{1}, \boldsymbol{\pi}\right)$ as

$$
\boldsymbol{\Delta}\left(\pi_{1}, \boldsymbol{\pi}\right)=\boldsymbol{\Delta}_{\text {comp }}\left(\pi_{1}, \boldsymbol{\pi} ; \gamma_{1}, \boldsymbol{\delta}\right) \boldsymbol{\Delta}\left(\gamma_{1}, \boldsymbol{\delta}\right)
$$

for one, and hence every, set of (strongly) G-regular related pairs (see Sections 4 and 12 of [S2]).

We will call $\mathbf{G}$ of quasi-split type if some $G^{j}$ is quasi-split and $\psi^{j}: G^{j} \rightarrow G^{*}$ is defined over $\mathbb{R}$. We will check in Lemma 12.6 that $j$ is then uniquely determined. Since $u^{j}$ lies in $Z\left(G_{s c}^{*}\right)$ we may as well take $u^{j}$ to be the identity, so that each $u^{k}$ is a cocycle. Thus the cochain $v^{k}$ above (and from the last section for the geometric case) is now a cocycle. Recall that the (geometric) factor $\Delta_{0}$ was defined in [LS1] for the case $G=G^{*}$ and $\psi=i d$, and the analogous spectral factor in [S2]. Each is a product $\Delta_{I} \Delta_{I I} \Delta_{I I I}$, where $\Delta_{I}$ and $\Delta_{I I}$ are the absolute terms already discussed (geometric $\Delta_{I I}$ includes old $\Delta_{I I I_{2}}$ ). We define extended factors $\boldsymbol{\Delta}_{0}$ in the same manner. All we need is an absolute version of relative $\Delta_{I I I}=\Delta_{I I I_{1}}$ in the geometric case and of relative $\Delta_{I I I}$ in the spectral case. Since each $v^{k}$ is a cocycle this is easy. To define the various terms we have chosen toral data for $\mathbf{G}$ (as well as $a$-data and for absolute $\Delta_{I}$, an $\mathbb{R}$-splitting of $G^{*}$ ). We may now pair the class of 
$v^{k}$ in $H^{1}\left(\Gamma, T_{s c}^{*}\right)$ with the element of $\pi_{0}\left(\left(T_{a d}^{\vee}\right)^{\Gamma}\right)$ provided by the endoscopic datum $s$ as in Section 3.4 of [LS1], and define $\Delta_{I I I}$ as the inverse of this pairing. Then

$$
\Delta_{I I I}\left(\gamma_{1}, \delta^{k}\right) / \Delta_{I I I}\left(\gamma_{1}^{\prime}, \delta^{k^{\prime}}\right)=\Delta_{I I I}\left(\gamma_{1}, \delta^{k} ; \gamma_{1}^{\prime}, \delta^{k^{\prime}}\right)
$$

and

$$
\Delta_{I I I}\left(\pi_{1}, \pi^{k}\right) / \Delta_{I I I}\left(\pi_{1}^{\prime}, \pi^{k^{\prime}}\right)=\Delta_{I I I}\left(\pi_{1}, \pi^{k} ; \pi_{1}^{\prime}, \pi^{k^{\prime}}\right),
$$

for all (strongly) G-regular related pairs. Set

$$
\boldsymbol{\Delta}_{0}=\Delta_{I} \Delta_{I I} \Delta_{I I I}
$$

Moreover, the geometric and spectral factors are compatible. The Whittaker normalizations (see [KS, S2] then also extend. We may use the $\mathbb{R}$-isomorphism $\psi^{j}$ to transport Whittaker data from $G^{*}$ to $G^{j}$, if we wish. Finally, we will see that there is no harm for the transfer theorem in replacing the endoscopic group $H_{1}$ by a $K$-group $\mathbf{H}_{1}$ of quasi-split type. Then in the spectral transfer factors, the representation $\pi_{1}$ may be replaced by any representation in its extended $L$-packet and will be denoted $\boldsymbol{\pi}_{1}$. Similarly, the element $\gamma_{1}$ in a geometric transfer factor is replaced by $\gamma_{1}$ in $\mathbf{H}_{1}(\mathbb{R})$.

\section{EXTENDED TRANSFER THEOREM AND ENDOSCOPIC CHARACTERS}

Our final step in preparation is to record an extension of the transfer theorem of S2 to $K$-groups. Extension of the geometric side was noted in A2. We may also state the theorem as a simultaneous version of Theorem 5.1 of [S2] for the various groups comprising the $K$-group.

We start with a group $G$ and inner twist $\psi$, and attach $K$-group G. Next, $(H, \mathcal{H}, s, \xi)$ is a set of endoscopic data for $G$. We attach $K$-group $\mathbf{H}$ to $H$, as well as a $z$-pair $\left(H_{1}, \xi_{1}\right)$ with character $\lambda_{1}$ on $Z_{1}(\mathbb{R})$, where $Z_{1}=\operatorname{Ker}\left(H_{1} \rightarrow H\right)$. We then construct a $z$-pair $\left(\mathbf{H}_{1}, \boldsymbol{\xi}_{1}\right)$ for the $K$-group $\mathbf{H}$ in the obvious manner, as well as attached character $\boldsymbol{\lambda}_{1}$ on $\mathbf{Z}_{1}(\mathbb{R})$, where $\mathbf{Z}_{1}=\operatorname{Ker}\left(\mathbf{H}_{1} \rightarrow \mathbf{H}\right)$. To save notation, we will assume each component of $\mathbf{Z}_{1}$ is $Z_{1}$ and each component of $\boldsymbol{\xi}_{1}$ is $\xi_{1}$, so that $\lambda_{1}$ consists of copies of the character $\lambda_{1}$. Also, Schwartz functions, Haar measures etc. are defined in the evident manner (see [A2]).

Lemma 6.1. Let $\mathbf{f}_{1} \in \mathcal{C}\left(\mathbf{H}_{1}(\mathbb{R}), \boldsymbol{\lambda}_{1}\right)$. Then $S t-\operatorname{Tr} \boldsymbol{\pi}_{1}\left(\mathbf{f}_{1}\right)=0$ for all tempered irreducible representations $\boldsymbol{\pi}_{1}$ such that $\boldsymbol{\pi}_{1}\left(\mathbf{z}_{1} \mathbf{h}_{1}\right)=\boldsymbol{\lambda}_{1}\left(\mathbf{z}_{1}\right) \pi\left(\mathbf{h}_{1}\right)$, for all $\mathbf{z} \in \mathbf{Z}_{1}(\mathbb{R})$ and $\mathbf{h}_{1} \in \mathbf{H}_{1}(\mathbb{R})$, if and only if $S O_{\gamma_{1}}\left(\mathbf{f}_{1}\right)=0$ for all strongly regular $\gamma_{1}$ in $\mathbf{H}_{1}(\mathbb{R})$.

Proof. We may use the stable transfer of orbital integrals to the quasi-split form S4] to rewrite $S O_{\gamma_{1}}\left(\mathbf{f}_{1}\right)$ as a stable orbital integral on $H_{1}(\mathbb{R})$. We then apply the

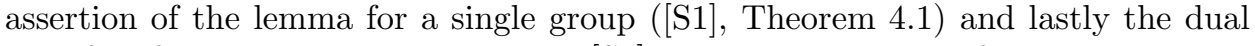
transfer of stable tempered characters [S4] to complete the proof.

Theorem 6.2 (Transfer theorem for $K$-groups). Let $\boldsymbol{\Delta}_{\text {geom }}$ and $\boldsymbol{\Delta}_{\text {spec }}$ be extended transfer factors with compatible normalization. Then for each $\mathbf{f} \in \mathcal{C}(\mathbf{G}(\mathbb{R}))$ there exists $\mathbf{f}_{1} \in \mathcal{C}\left(\mathbf{H}_{1}(\mathbb{R}), \boldsymbol{\lambda}_{1}\right)$ such that

$$
S O_{\boldsymbol{\gamma}_{1}}\left(\mathbf{f}_{1}\right)=\sum_{\boldsymbol{\delta}, \mathrm{conj}} \boldsymbol{\Delta}_{\text {geom }}\left(\boldsymbol{\gamma}_{1}, \boldsymbol{\delta}\right) O_{\delta}(\mathbf{f})
$$


for all strongly $\mathbf{G}$-regular $\gamma_{1}$ in $\mathbf{H}_{1}(\mathbb{R})$. Moreover, there is a dual transfer of stable tempered characters given by

$$
S t-\operatorname{Tr} \boldsymbol{\pi}_{1}\left(\mathbf{f}_{1}\right)=\sum_{\boldsymbol{\pi}, t e m p} \boldsymbol{\Delta}_{\text {spec }}\left(\boldsymbol{\pi}_{1}, \boldsymbol{\pi}\right) \operatorname{Tr} \boldsymbol{\pi}(\mathbf{f})
$$

for all tempered irreducible representations $\boldsymbol{\pi}_{1}$ of $\mathbf{H}_{1}(\mathbb{R})$ which transform under $\mathbf{Z}_{1}(\mathbb{R})$ according to $\boldsymbol{\lambda}_{1}$. Conversely, if $\mathbf{f} \in \mathcal{C}(\mathbf{G}(\mathbb{R}))$ and $\mathbf{f}_{1} \in \mathcal{C}\left(\mathbf{H}_{1}(\mathbb{R}), \boldsymbol{\lambda}_{1}\right)$ satisfy

$$
S t-\operatorname{Tr} \boldsymbol{\pi}_{1}\left(\mathbf{f}_{1}\right)=\sum_{\boldsymbol{\pi}, t e m p} \boldsymbol{\Delta}_{\text {spec }}\left(\boldsymbol{\pi}_{1}, \boldsymbol{\pi}\right) \operatorname{Tr} \boldsymbol{\pi}(\mathbf{f})
$$

for all tempered irreducible representations $\boldsymbol{\pi}_{1}$ of $\mathbf{H}_{1}(\mathbb{R})$ which transform under $\mathbf{Z}_{1}(\mathbb{R})$ according to $\boldsymbol{\lambda}_{1}$ then

$$
S O_{\gamma_{1}}\left(\mathbf{f}_{1}\right)=\sum_{\boldsymbol{\delta}, \mathrm{conj}} \boldsymbol{\Delta}_{g e o m}\left(\boldsymbol{\gamma}_{1}, \boldsymbol{\delta}\right) O_{\boldsymbol{\delta}}(\mathbf{f})
$$

for all strongly $\mathbf{G}$-regular $\gamma_{1}$ in $\mathbf{H}_{1}(\mathbb{R})$.

Proof. First of all, we have the transfer theorem of [S2] for the forms $G^{j}$ using the normalizations $\Delta^{j}$ provided by the $K$-group normalizations of Sections 4 and 5 . Suppose $\mathbf{f}=\left\{f^{j}\right\}$ and for each $j$ choose $f_{1}^{j} \in \mathcal{C}\left(H_{1}(\mathbb{R}), \lambda_{1}\right)$ such that

$$
S O_{\gamma_{1}}\left(f_{1}^{j}\right)=\sum_{\delta^{j}, \text { conj }} \Delta_{\text {geom }}^{j}\left(\gamma_{1}, \delta^{j}\right) O_{\delta^{j}}\left(f^{j}\right) .
$$

Then we sum these identities over $j$ and set $f_{1}=\sum_{j} f_{1}^{j}$. Regarding $f_{1}$ as $\mathbf{f}_{1} \in$ $\mathcal{C}\left(\mathbf{H}_{1}(\mathbb{R}), \boldsymbol{\lambda}_{1}\right)$, we obtain the geometric transfer. Suppose now $\mathbf{f}$ and $\mathbf{f}_{1}$ have matching orbital integrals. By Lemma $6.1, S t-\operatorname{Tr} \boldsymbol{\pi}_{1}\left(\mathbf{f}_{1}\right)$ is independent of the choice for $\mathbf{f}_{1}$. Thus we may take $\mathbf{f}_{1}$ to be $f_{1}$ as above. Since we have

$$
\operatorname{St}-\operatorname{Tr} \pi_{1}\left(f_{1}^{j}\right)=\sum_{\pi^{j}, \text { temp }} \Delta_{\text {spec }}^{j}\left(\pi_{1}, \pi^{j}\right) \operatorname{Tr} \pi^{j}\left(f^{j}\right)
$$

for all $j$, the spectral matching follows. The converse statement follows as for a single group $G$ ( S1], Section 16 ) using Lemma 6.1. This completes the proof.

As in Section 3 for a single group, we call the tempered virtual character

$$
\mathbf{f} \rightarrow \operatorname{St}-\operatorname{Tr} \boldsymbol{\pi}_{1}\left(\mathbf{f}_{1}\right)
$$

an endoscopic character on $\mathbf{G}(\mathbb{R})$. The results and comments of Sections 2 and 3 generalize immediately to the present setting.

\section{INVERSION AND ADJOINT RELATIONS}

Let $\boldsymbol{\pi}$ be a tempered irreducible admissible representation of $\mathbf{G}(\mathbb{R})$. Our plan is to recover $\operatorname{Tr} \boldsymbol{\pi}(\mathbf{f})$ from the identities (for various $\mathbf{H}_{1}, \boldsymbol{\pi}_{1}$ )

$$
S t-\operatorname{Tr} \boldsymbol{\pi}_{1}\left(\mathbf{f}_{1}\right)=\sum_{\boldsymbol{\pi}^{\prime}, \text { temp }} \boldsymbol{\Delta}_{\text {spec }}\left(\boldsymbol{\pi}_{1}, \boldsymbol{\pi}^{\prime}\right) \operatorname{Tr} \boldsymbol{\pi}^{\prime}(\mathbf{f})
$$

of the transfer theorem. We do this by establishing an explicit set of adjoint relations

$$
\sum_{\boldsymbol{\pi}_{1}, \text { temp }} \overline{\boldsymbol{\Delta}\left(\boldsymbol{\pi}_{1}, \boldsymbol{\pi}\right)} \boldsymbol{\Delta}\left(\boldsymbol{\pi}_{1}, \boldsymbol{\pi}^{\prime}\right)=n\left(\boldsymbol{\pi}, \boldsymbol{\pi}^{\prime}\right) .
$$

In this summation $\sum_{\boldsymbol{\pi}_{1}, \text { temp }}$ over certain ( $L$-packets of) representations $\boldsymbol{\pi}_{1}$ of endoscopic groups, yet to be defined precisely, there will be only finitely many 
nonzero terms in which our given $\boldsymbol{\pi}$ appears. Note that by the definition of $\boldsymbol{\Delta}$, if $\boldsymbol{\Delta}\left(\boldsymbol{\pi}_{1}, \boldsymbol{\pi}\right)$ is nonzero then

$$
\overline{\boldsymbol{\Delta}\left(\boldsymbol{\pi}_{1}, \boldsymbol{\pi}\right)} \boldsymbol{\Delta}\left(\boldsymbol{\pi}_{1}, \boldsymbol{\pi}^{\prime}\right)
$$

is nonzero exactly when $\boldsymbol{\pi}^{\prime}$ lies in the same $L$-packet as $\boldsymbol{\pi}$. We will see that the sum $n\left(\boldsymbol{\pi}, \boldsymbol{\pi}^{\prime}\right)$ is zero unless $\boldsymbol{\pi}^{\prime}=\boldsymbol{\pi}$. Evidently $n(\boldsymbol{\pi}, \boldsymbol{\pi})$ is the product of $\|\boldsymbol{\Delta}\|^{2}$ (see Section 12 of [S2]) with the number $n(\boldsymbol{\pi})$ of nonzero terms $\boldsymbol{\Delta}\left(\boldsymbol{\pi}_{1}, \boldsymbol{\pi}\right)$ in the summation $\sum_{\boldsymbol{\pi}_{1}, \text { temp }}$. The summation $\sum_{\boldsymbol{\pi}^{\prime}, \text { temp }}$ from the transfer theorem similarly has only finitely many nonzero terms (corresponding to $\boldsymbol{\pi}^{\prime}$ from a single $L$-packet) in which given $\boldsymbol{\pi}_{1}$ appears.

Once the adjoint relations are established, we have just to multiply the identity from the transfer theorem by the factor

$$
\frac{\overline{\boldsymbol{\Delta}\left(\boldsymbol{\pi}_{1}, \boldsymbol{\pi}\right)}}{n(\boldsymbol{\pi})\|\boldsymbol{\Delta}\|^{2}}
$$

then apply the summation $\sum_{\boldsymbol{\pi}_{1}, \text { temp }}$ and reverse the order of the summations, to obtain

$$
\operatorname{Tr} \boldsymbol{\pi}(\mathbf{f})=\frac{1}{n(\boldsymbol{\pi})\|\boldsymbol{\Delta}\|^{2}} \sum_{\boldsymbol{\pi}_{1}, t e m p} \overline{\boldsymbol{\Delta}\left(\boldsymbol{\pi}_{1}, \boldsymbol{\pi}\right)} \operatorname{St}-\operatorname{Tr} \boldsymbol{\pi}_{1}\left(\mathbf{f}_{1}\right),
$$

the desired inversion formula.

\section{Lemma 7.1.}

$$
\frac{\overline{\Delta\left(\pi_{1}, \pi\right)}}{\|\Delta\|^{2}}=\Delta\left(\pi_{1}, \pi\right)^{-1}
$$

for all tempered related pairs $\left(\boldsymbol{\pi}_{1}, \boldsymbol{\pi}\right)$.

Proof. For any $G$-regular related pair $\left(\boldsymbol{\pi}_{1}, \boldsymbol{\pi}\right)$ we have

$$
\left|\boldsymbol{\Delta}\left(\boldsymbol{\pi}_{1}, \boldsymbol{\pi}\right)\right|=\|\boldsymbol{\Delta}\|,
$$

and so the assertion is clear in that case. We extend the formula to all tempered pairs by coherent continuation (see the formulas of Section 14 of [S2]).

We may thus rewrite the inversion as

$$
\operatorname{Tr} \boldsymbol{\pi}(\mathbf{f})=\frac{1}{n(\boldsymbol{\pi})} \sum_{\boldsymbol{\pi}_{1}, t e m p} \boldsymbol{\Delta}\left(\boldsymbol{\pi}_{1}, \boldsymbol{\pi}\right)^{-1} \operatorname{St}-\operatorname{Tr} \boldsymbol{\pi}_{1}\left(\mathbf{f}_{1}\right) .
$$

Notice that the inversion formula will be independent of the normalization of transfer factors. More precisely:

Lemma 7.2. A change in normalization of the compatible transfer factors $\boldsymbol{\Delta}_{\text {geom }}$ and $\boldsymbol{\Delta}_{\text {spec }}=\boldsymbol{\Delta}$ does not change

$$
\begin{aligned}
& \frac{1}{\|\boldsymbol{\Delta}\|^{2}} \overline{\boldsymbol{\Delta}\left(\boldsymbol{\pi}_{1}, \boldsymbol{\pi}\right)} \operatorname{St}-\operatorname{Tr} \boldsymbol{\pi}_{1}\left(\mathbf{f}_{1}\right) \\
& =\boldsymbol{\Delta}\left(\boldsymbol{\pi}_{1}, \boldsymbol{\pi}\right)^{-1} \operatorname{St}-\operatorname{Tr} \boldsymbol{\pi}_{1}\left(\mathbf{f}_{1}\right) .
\end{aligned}
$$

Proof. We have to multiply $\boldsymbol{\Delta}_{\text {geom }}$ and $\boldsymbol{\Delta}_{\text {spec }}$ by the same constant. Then $\mathbf{f}_{1}$ may be replaced by the same multiple of itself in the new transfer theorem, and so the lemma is proved.

The following begins the proof of the inversion. See [A2 (Section 3) for the geometric analogue. 
Lemma 7.3. Assume that $\left(\boldsymbol{\pi}_{1}, \boldsymbol{\pi}\right)$ and $\left(\boldsymbol{\pi}_{1}, \boldsymbol{\pi}^{\prime}\right)$ are $G$-regular related pairs. Then

$$
\frac{1}{\|\boldsymbol{\Delta}\|^{2}} \overline{\boldsymbol{\Delta}\left(\boldsymbol{\pi}_{1}, \boldsymbol{\pi}\right)} \boldsymbol{\Delta}\left(\boldsymbol{\pi}_{1}, \boldsymbol{\pi}^{\prime}\right)=\boldsymbol{\Delta}_{I I I}\left(\boldsymbol{\pi}_{1}, \boldsymbol{\pi} ; \boldsymbol{\pi}_{1}, \boldsymbol{\pi}^{\prime}\right)
$$

provided the same toral data are chosen for each of $\left(\boldsymbol{\pi}_{1}, \boldsymbol{\pi}\right)$ and $\left(\boldsymbol{\pi}_{1}, \boldsymbol{\pi}^{\prime}\right)$.

Proof.

$$
\frac{1}{\|\boldsymbol{\Delta}\|^{2}} \overline{\boldsymbol{\Delta}\left(\boldsymbol{\pi}_{1}, \boldsymbol{\pi}\right)} \boldsymbol{\Delta}\left(\boldsymbol{\pi}_{1}, \boldsymbol{\pi}^{\prime}\right)=\boldsymbol{\Delta}\left(\boldsymbol{\pi}_{1}, \boldsymbol{\pi}\right)^{-1} \boldsymbol{\Delta}\left(\boldsymbol{\pi}_{1}, \boldsymbol{\pi}^{\prime}\right)
$$

is just the canonical factor

$$
\boldsymbol{\Delta}\left(\boldsymbol{\pi}_{1}, \boldsymbol{\pi} ; \boldsymbol{\pi}_{1}, \boldsymbol{\pi}^{\prime}\right) .
$$

Under the assumption on the toral data, we have

$$
\boldsymbol{\Delta}_{I}\left(\boldsymbol{\pi}_{1}, \boldsymbol{\pi} ; \boldsymbol{\pi}_{1}, \boldsymbol{\pi}^{\prime}\right) \boldsymbol{\Delta}_{I I}\left(\boldsymbol{\pi}_{1}, \boldsymbol{\pi} ; \boldsymbol{\pi}_{1}, \boldsymbol{\pi}^{\prime}\right)=1
$$

(and the terms themselves are trivial if we further require the same $a$-data for each pair). Thus we obtain

$$
\boldsymbol{\Delta}_{I I I}\left(\boldsymbol{\pi}_{1}, \boldsymbol{\pi} ; \boldsymbol{\pi}_{1}, \boldsymbol{\pi}^{\prime}\right),
$$

which completes the proof.

Corollary 7.4. In the setting of the lemma, the term $\boldsymbol{\Delta}_{I I I}\left(\boldsymbol{\pi}_{1}, \boldsymbol{\pi} ; \boldsymbol{\pi}_{1}, \boldsymbol{\pi}^{\prime}\right)$ is canonical.

To specify the summation $\sum_{\boldsymbol{\pi}_{1}, \text { temp }}$ for a given tempered representation $\boldsymbol{\pi}$ of $\mathbf{G}(\mathbb{R})$ we return to Langlands' original constructions. Suppose that the Langlands parameter for $\pi$ is the $G^{\vee}$-conjugacy class $\varphi$ of $\varphi: W_{\mathbb{R}} \rightarrow{ }^{L} G$, and set $S_{\varphi}=$ $\operatorname{Cent}\left(\varphi\left(W_{\mathbb{R}}\right), G^{\vee}\right)$. We will make use of $S_{\varphi}^{a d}$, the image of $S_{\varphi}$ in $G_{a d}^{\vee}=G^{\vee} / Z\left(G^{\vee}\right)$, and of $S_{\varphi}^{s c}$ by which we will mean the inverse image of $S_{\varphi}^{a d}$ in $G_{s c}^{\vee}$. Notice that if $\varphi$ is regular, then $S_{\varphi}$ is just the set of $\varphi(1 \times \sigma)$-fixed points in the maximal torus $\operatorname{Cent}\left(\varphi\left(\mathbb{C}^{\times}\right), G^{\vee}\right)$, and so it is abelian. For all tempered $\varphi$ we set

$$
\mathbb{S}_{\varphi}^{a d}=\pi_{0}\left(S_{\varphi}^{a d}\right) \simeq S_{\varphi} / S_{\varphi}^{0} Z\left(G^{\vee}\right)^{\Gamma} .
$$

This group was denoted $\mathbb{S}_{\varphi}$ in Section 5 of [S3]. Also set $\mathbb{S}_{\varphi}^{s c}=\pi_{0}\left(S_{\varphi}^{s c}\right)$.

Keeping in mind Arthur's conjecture of [A1], we work with a semisimple element $s_{s c}$ of $S_{\varphi}^{s c}$ instead of an element of $S_{\varphi}$, and now always use $s$ to denote the image of $s_{s c}$ in $G^{\vee}$. Thus $s$ lies in $S_{\varphi}$ only up to an element of $Z\left(G^{\vee}\right)$, but that will be sufficient to attach endoscopic data

$$
\mathfrak{e}\left(s_{s c}\right)=\mathfrak{e}(s)=\left(H^{(s)}, \mathcal{H}^{s}, s, \xi^{s}\right)
$$

in the usual manner. Recall that $\mathcal{H}^{s}$ is the subgroup of ${ }^{L} G$ generated by $\operatorname{Cent}\left(s, G^{\vee}\right)^{0}$ and the image of $\varphi$, while $\xi^{s}$ is inclusion and $H^{(s)}$ is a dual quasi-split group. We noted the requirements (i)-(iv) for endoscopic data in Section 2. It is immediate that these are each satisfied by $\mathfrak{e}(s)$. We have used the notation $H^{(s)}$ to preserve $H^{s}$ for $z$-extensions.

Fix some attached $z$-pair $\left(H^{s}, \xi_{1}^{s}\right)$ for $\mathfrak{e}(s)$. By construction, $\varphi\left(W_{\mathbb{R}}\right)$ lies in $\mathcal{H}^{s}$ and so we have a well-defined homomorphism $\varphi^{s}=\xi_{1}^{s} \circ \varphi$ representing an element $\boldsymbol{\varphi}^{s}$ of $\Phi_{\text {temp }}\left(\mathbf{H}^{s}, \boldsymbol{\lambda}_{1}^{s}\right)$, where $\boldsymbol{\lambda}_{1}^{s}$ is the character attached to the $z$-pair $\left(H^{s}, \xi_{1}^{s}\right)$. Of course, $\varphi^{s}$ maps to $\varphi$ under

$$
\Phi_{\text {temp }}\left(\mathbf{H}^{s}, \boldsymbol{\lambda}_{1}^{s}\right) \rightarrow \Phi_{\text {temp }}(\mathbf{G}) .
$$

We write $\boldsymbol{\pi}^{s}$ for a representation of $\mathbf{H}^{s}(\mathbb{R})$ with parameter $\boldsymbol{\varphi}^{s}$. 
In the summation $\sum_{\boldsymbol{\pi}_{1}, \text { temp }}$ we will take $\boldsymbol{\pi}_{1}=\boldsymbol{\pi}^{s}$. Because we find that

$$
\frac{1}{\|\boldsymbol{\Delta}\|^{2}} \overline{\Delta\left(\boldsymbol{\pi}^{s}, \boldsymbol{\pi}\right)} \boldsymbol{\Delta}\left(\boldsymbol{\pi}^{s}, \boldsymbol{\pi}^{\prime}\right)
$$

depends only on the image $s_{a d}$ of $s_{s c}$ under $S_{\varphi}^{s c} \rightarrow S_{\varphi}^{a d} \rightarrow \mathbb{S}_{\varphi}^{a d}$ (Theorem 7.5 (i) below), we may take the summation over $\mathbb{S}_{\varphi}^{a d}$ or, more precisely, over semisimple representatives $s_{s c}$ for $\mathbb{S}_{\varphi}^{s c} / \operatorname{Ker}\left(\mathbb{S}_{\varphi}^{s c} \rightarrow \mathbb{S}_{\varphi}^{a d}\right)$, and then $n(\boldsymbol{\pi})$ will be the order of $\mathbb{S}_{\varphi}^{a d}$.

For semisimple $s_{s c} \in S_{\varphi}^{s c}$ and $\boldsymbol{\pi}^{\prime}$ in the $L$-packet of $\boldsymbol{\pi}$, define

$$
\Phi\left(s_{s c}\right)=\Phi_{\boldsymbol{\pi}, \boldsymbol{\pi}^{\prime}}\left(s_{s c}\right)=\frac{1}{\|\boldsymbol{\Delta}\|^{2}} \overline{\boldsymbol{\Delta}\left(\boldsymbol{\pi}^{s}, \boldsymbol{\pi}\right)} \boldsymbol{\Delta}\left(\boldsymbol{\pi}^{s}, \boldsymbol{\pi}^{\prime}\right) .
$$

By Lemma 7.1,

$$
\Phi_{\boldsymbol{\pi}, \boldsymbol{\pi}^{\prime}}\left(s_{s c}\right)=\boldsymbol{\Delta}\left(\boldsymbol{\pi}^{s}, \boldsymbol{\pi}\right)^{-1} \boldsymbol{\Delta}\left(\boldsymbol{\pi}^{s}, \boldsymbol{\pi}^{\prime}\right)
$$

and so

$$
\Phi_{\boldsymbol{\pi}, \boldsymbol{\pi}^{\prime}}\left(s_{s c}\right) \Phi_{\boldsymbol{\pi}^{\prime}, \boldsymbol{\pi}^{\prime \prime}}\left(s_{s c}\right)=\Phi_{\boldsymbol{\pi}, \boldsymbol{\pi}^{\prime \prime}}\left(s_{s c}\right)
$$

for all $\boldsymbol{\pi}, \boldsymbol{\pi}^{\prime}, \boldsymbol{\pi}^{\prime \prime}$ in the same $L$-packet.

\section{Theorem 7.5.}

(i) $\Phi\left(s_{s c}\right)$ depends only on the image $s_{a d}$ of $s_{s c}$ under $S_{\varphi}^{s c} \rightarrow \mathbb{S}_{\varphi}^{s c} \rightarrow \mathbb{S}_{\varphi}^{a d}$.

(ii) $s_{a d} \rightarrow \Phi\left(s_{s c}\right)$ is a character on $\mathbb{S}_{\varphi}^{a d}$ which is nontrivial unless $\boldsymbol{\pi}=\boldsymbol{\pi}^{\prime}$.

(iii) All characters on $\mathbb{S}_{\varphi}^{a d}$ are so obtained.

This theorem gathers up results from Section 5 of $[\mathbf{S} 3$ into the $K$-group setting. For the proof we will recall what we need from [S3] step by step in the next three sections, and integrate this with the needed properties of $K$-groups we have discussed in Sections 4 and 5.

Corollary 7.6. Summing over semisimple representatives $s_{s c}$ for

$$
\mathbb{S}_{\varphi}^{s c} / \operatorname{Ker}\left(\mathbb{S}_{\varphi}^{s c} \rightarrow \mathbb{S}_{\varphi}^{a d}\right) \simeq \mathbb{S}_{\varphi}^{a d}
$$

we obtain

$$
\frac{1}{\|\boldsymbol{\Delta}\|^{2}} \sum_{\mathbf{S}_{s c}} \overline{\boldsymbol{\Delta}\left(\boldsymbol{\pi}^{s}, \boldsymbol{\pi}\right)} \boldsymbol{\Delta}\left(\boldsymbol{\pi}^{s}, \boldsymbol{\pi}^{\prime}\right)=\left|\mathbb{S}_{\varphi}^{a d}\right| K r\left(\boldsymbol{\pi}, \boldsymbol{\pi}^{\prime}\right),
$$

where $K r$ denotes the Kronecker delta function.

Corollary 7.7. For each tempered irreducible admissible representation $\boldsymbol{\pi}$ of $\mathbf{G}(\mathbb{R})$ we have

$$
\begin{aligned}
\operatorname{Tr} \boldsymbol{\pi}(\mathbf{f}) & =\frac{1}{n(\boldsymbol{\pi})\|\boldsymbol{\Delta}\|^{2}} \sum_{\mathbf{s}_{s c}} \overline{\boldsymbol{\Delta}\left(\boldsymbol{\pi}^{s}, \boldsymbol{\pi}\right)} \operatorname{St}-\operatorname{Tr} \boldsymbol{\pi}^{s}\left(\mathbf{f}^{s}\right) \\
& =\frac{1}{n(\boldsymbol{\pi})} \sum_{\mathbf{s}_{s c}} \boldsymbol{\Delta}\left(\boldsymbol{\pi}^{s}, \boldsymbol{\pi}\right)^{-1} \operatorname{St}-\operatorname{Tr} \boldsymbol{\pi}^{s}\left(\mathbf{f}^{s}\right),
\end{aligned}
$$

where $n(\boldsymbol{\pi})=\left|\mathbb{S}_{\varphi}^{a d}\right|$ is the cardinality of the L-packet of $\boldsymbol{\pi}$.

\section{Lemma 7.8.}

(i) Each summand in the inversion formula of Corollary 7.7 is independent of the choice of representative for the Langlands parameter $\varphi$ of $\pi$.

(ii) Also, each summand is independent of the choice of z-pair $\left(H_{1}^{s}, \xi_{1}^{s}\right)$ attached to the endoscopic data $\mathfrak{e}(s)$. 
Proof. Applying Lemma 2.4 to the construction of $\mathfrak{e}(s)$ shows that the terms on the right side are unchanged when $\varphi$ is replaced by another representative Int $g \circ \varphi$, $g \in G^{\vee}$. For a change in $z$-pair we apply Lemma 3.1.

\section{Regular ElLiptic CASE}

We now prove Theorem 7.5 in the case that $\boldsymbol{\pi}$ is elliptic as well as regular, i.e., $\boldsymbol{\pi}$ is a discrete series representation. We apply the $K$-group setting to the example of Section 5.2 of [S3]. See Section 3 of [A2] for a parallel argument on the geometric side. In particular, we see how $\mathcal{E}(T)$ replaces $\mathcal{D}(T)$ in the parametrization of discrete $L$-packets (recall the small examples of Section 4 ).

Thus $\mathrm{G}$ is cuspidal and $\varphi=\varphi(\pi)$ is discrete, i.e. the image of any representative $\varphi$ for $\varphi$ is contained in no proper parabolic subgroup of ${ }^{L} G$. Let $\mathcal{T}$ be the maximal torus $\operatorname{Cent}\left(\varphi\left(\mathbb{C}^{\times}\right), G^{\vee}\right)$ in $G^{\vee}$. Then $S_{\varphi}$ consists of the elements of $\mathcal{T}$ fixed by the automorphism dual to the Galois action of a Cartan subgroup $\mathbf{T}$ compact modulo the center of $\mathbf{G}$. Thus the nontrivial elements of $S_{\varphi}^{a d}=\mathbb{S}_{\varphi}^{a d}$ are exactly the elements of order two in the image $\mathcal{T}_{a d}$ of $\mathcal{T}$ in $G_{a d}^{\vee}$. Then $S_{\varphi}^{s c}=\mathbb{S}_{\varphi}^{s c}$, an extension of $\mathbb{S}_{\varphi}^{a d}$ by $Z\left(G_{s c}^{\vee}\right)$, is a subgroup of the inverse image $\mathcal{T}_{s c}$ of $\mathcal{T}_{a d}$ in $G_{s c}^{\vee}$, and so is abelian as well as finite.

For each $s_{s c}$ in $S_{\varphi}^{s c}$, the parameter $\varphi^{s}$ must be $G$-regular and discrete, so that $\boldsymbol{\pi}^{s}$ is also a discrete series representation. The attached set $\mathfrak{e}(s)$ of endoscopic data is thus elliptic, and $\mathbf{H}^{s}$ shares each Cartan subgroup $\mathbf{T}$ of $\mathbf{G}$ that is compact modulo the center of $\mathbf{G}$.

Before continuing, we recall representatives $\varphi=\varphi(\mu, \lambda)$ for $\varphi$ and $\varphi^{s}=\varphi\left(\mu^{s}, \lambda^{s}\right)$ for $\varphi^{s}$. We have fixed a splitting $s p l_{G^{\vee}}=\left(\mathcal{B}, \mathcal{T},\left\{X_{\alpha^{\vee}}\right\}\right)$. Let $\iota$ be one half the sum of the roots of $\mathcal{T}$ in $\mathcal{B}$. Then according to [L2] there is a pair $\mu, \lambda \in X_{*}(\mathcal{T}) \otimes \mathbb{C}$ satisfying

$$
\frac{1}{2}\left(\mu-\sigma_{\mathbf{T}} \mu\right)-\iota+\left(\lambda+\sigma_{\mathbf{T}} \lambda\right) \in X_{*}(\mathcal{T})
$$

and with $\mu$ (thus integral) regular and $\mathcal{B}$-dominant, with the property that a representative $\varphi=\varphi(\mu, \lambda)$ is given by

$$
\varphi(z \times 1)=z^{\mu} \bar{z}^{\sigma_{\mathbf{T}}(\mu)}
$$

for $z \in \mathbb{C}^{\times}$, and

$$
\varphi(1 \times \sigma)=e^{2 \pi i \lambda} n\left(\sigma_{\mathbf{T}}\right) \times(1 \times \sigma),
$$

with $n\left(\sigma_{\mathbf{T}}\right)$ the element of $G^{\vee}$ attached by $\left\{X_{\alpha^{\vee}}\right\}$ to the Weyl group element $w\left(\sigma_{\mathbf{T}}\right)$, where $\sigma_{\mathbf{T}}$ acts as $w\left(\sigma_{\mathbf{T}}\right) \circ \sigma$ on $\mathcal{T}$, as in Section 2.1 of [LS1]. Here $\mu$ is determined uniquely, while $\lambda$ is determined only modulo

$$
X_{*}(\mathcal{T})+\left\{\nu-\sigma_{T} \nu: \nu \in X_{*}(\mathcal{T}) \otimes \mathbb{C}\right\},
$$

and so $\varphi$ is determined uniquely up to $\mathcal{T}$-conjugacy.

Consider the embeddings $\left(\xi_{s}, \xi_{s}^{1}\right)$ attached to the endoscopic data $\mathfrak{e}(s)$. Recall that $\xi_{s}$ is the inclusion map of $\mathcal{H}^{s}$ in ${ }^{L} G$, and $\xi_{s}^{1}$ embeds $\mathcal{H}^{s}$ in the $L$-group ${ }^{L} H^{s}$ of the $z$-extension $H^{s}$. There are some harmless assumptions we make: that $\mathcal{T}$ is the maximal torus and $\mathcal{B} \cap\left(H^{(s)}\right)^{\vee}$ is the Borel subgroup specified by our chosen $\Gamma$-splitting $s p l^{s}$ for $\left(H^{(s)}\right)^{\vee}$, that $\left(H^{(s)}\right)^{\vee}$ is a subgroup of $\left(H^{s}\right)^{\vee}$, and that the splitting we use for $\left(H^{s}\right)^{\vee}$ is the natural extension $s p l_{1}^{s}$ of $s p l^{s}$. We now denote by $\left(\mu^{* s}, \lambda^{* s}\right)$ the pair $\left(\mu^{*}, \lambda^{*}\right)$ attached in Section 11 of [S1] to the embeddings $\left(\xi_{s}, \xi_{s}^{1}\right)$. 
Each of $\mu^{* s}, \lambda^{* s}$ lie in the space $X_{*}\left(\mathcal{T}_{1}\right) \otimes \mathbb{C}$ containing $X_{*}(\mathcal{T}) \otimes \mathbb{C}$. We see then that the parameter $\varphi^{s}$ for $H_{1}^{s}$ has data $\left(\mu^{s}, \lambda^{s}\right)$ relative to $s p l_{1}^{s}$, where

$$
\mu^{s}=\mu-\mu^{* s} \text { and } \lambda^{s}=\lambda-\lambda^{* s} \text {. }
$$

Given $s_{s c}$ in $S_{\varphi}^{s c}$ and thus the endoscopic data $\mathfrak{e}(s)$, we now fix toral data for a fundamental maximal torus $\mathbf{T}$ over $\mathbb{R}$ in $\mathbf{G}$. This includes, in particular, a fundamental maximal torus $T^{*}$ in $G^{*}$. Because $T^{*}$ is compact modulo center, we may insist that the underlying isomorphism $\mathcal{T} \rightarrow\left(T^{*}\right)^{\vee}$ is independent of the choice of $s_{s c}$. For our chosen representative $\varphi=\varphi(\mu, \lambda)$, we have $S_{\varphi}$ contained in $\mathcal{T}$ and so $\mathcal{T} \rightarrow\left(T^{*}\right)^{\vee}$ provides an isomorphism of $S_{\varphi}$ with the invariants in $\left(T^{*}\right)^{\vee}$ for the Galois action on $T^{*}$.

Proof of Theorem 7.5 ( $\varphi$ discrete). By Lemma 7.3, we have

$$
\Phi\left(s_{s c}\right)=\boldsymbol{\Delta}_{I I I}\left(\boldsymbol{\pi}^{s}, \boldsymbol{\pi} ; \boldsymbol{\pi}^{s}, \boldsymbol{\pi}^{\prime}\right) .
$$

By definition (following Section 10 of [S2] and Section 3.4 of [LS1]),

$$
\boldsymbol{\Delta}_{I I I}\left(\boldsymbol{\pi}^{s}, \boldsymbol{\pi} ; \boldsymbol{\pi}^{s}, \boldsymbol{\pi}^{\prime}\right)=\left\langle\left(v^{-1}, v^{\prime}\right)_{U}, s_{U}\right\rangle,
$$

where $\langle$,$\rangle denotes the Tate-Nakayama pairing for the torus U=U\left(T^{*}, T^{*}\right)$. Since $s_{U}$ depends only on $s_{a d}$ we conclude that $\Phi\left(s_{s c}\right)$ depends only on $s_{a d}$ also, and since $s_{a d} \rightarrow s_{U}$ is a homomorphism we see that $s_{a d} \rightarrow \Phi\left(s_{s c}\right)$ is a character.

Next, recall that the Tate-Nakayama pairing identifies $\mathbb{S}_{\varphi}^{a d}=S_{\varphi}^{a d} \backsim S_{\varphi} / Z\left(G^{\vee}\right)^{\Gamma}$ as the dual of $\mathcal{E}\left(T^{*}\right)$. Fix $\boldsymbol{\pi}$ and consider the characters $\Phi_{\boldsymbol{\pi}, \boldsymbol{\pi}^{\prime}}=\Phi_{\boldsymbol{\pi}^{\prime}}$ as $\boldsymbol{\pi}^{\prime}$ varies. It remains to show that we get $\left|\mathcal{E}\left(T^{*}\right)\right|$ distinct characters this way, with $\Phi_{\boldsymbol{\pi}}$ is trivial. Observe that, as in [A2, the product $v^{-1} v^{\prime}$ is a 1-cocycle of $\Gamma$ in $T_{s c}^{*}$ which we may map to $T^{*}$, thus defining an element of $\mathcal{E}\left(T^{*}\right)$. We may evaluate $\Phi_{\boldsymbol{\pi}^{\prime}}\left(s_{a d}\right)$ as $\left\langle v^{-1} v^{\prime}, s_{a d}\right\rangle$. So it is clear (again) that $\Phi_{\boldsymbol{\pi}} \equiv 1$.

Recall our notation associated with the $K$-group $\mathbf{G}$ in Section 4 . The chosen toral data provide $\mathbf{T}=\bigsqcup_{j} T^{j}$ as well as $T^{*}$. There is no harm in assuming that $\psi^{-1}=\left(\psi^{1}\right)^{-1}$ maps $T^{*}$ to $T=T^{1}$ over $\mathbb{R}$. Since we plan to work in $T^{1}$ we use $\psi^{1}$ to identify the two tori and suppress it in notation. Notice, in particular, that $\mathcal{E}\left(T^{*}\right)=\mathcal{E}\left(T^{1}\right)$. We may further assume that the cocycles $x_{s c}^{j}$, and so also the cochains $u^{j}$, each take values in $T_{s c}^{1}$ (see Section 10 of [K1]) and then that the isomorphisms $\psi^{j}: T^{1} \rightarrow T^{j}$ are part of the chosen toral data.

Recall also the notation of Sections $7 \mathrm{~b}$ and 10 of [S2 for discrete series representations (for given toral data). If $\boldsymbol{\pi}^{\prime}$ is a representation of $G^{j}$, then we write $\pi^{j}=\pi^{j}(w)$, for some $w$ in $\mathcal{D}\left(T^{j}\right)$. From $\boldsymbol{\pi}=\pi^{j}(1)$ and the various $\boldsymbol{\pi}^{\prime}=\pi^{j}(w)$ we get $\left|\mathcal{D}\left(T^{j}\right)\right|$ distinct characters $\Phi_{\boldsymbol{\pi}^{\prime}}$ in the usual manner [S3] (see also the paragraph before Remark 7.1 of [S2]).

There is no harm in assuming now that the fixed $\boldsymbol{\pi}$ is $\pi^{1}(1)$. Observe that if $\boldsymbol{\pi}^{\prime}=$ $\pi^{j}(1)$, then

$$
v^{-1} v^{\prime}=\left(u^{1}\right)^{-1} u^{j}=x_{s c}^{j}
$$

up to coboundaries in $T_{s c}^{1}$. More generally, we may estabish a bijection between $\mathcal{D}\left(T^{j}\right)$ and the classes in $\mathcal{E}\left(T^{1}\right)$ which map to the class of $x^{j}$ in $H(\Gamma, G)$. For this, we check that if $g \in G_{s c}^{1}$ and / denotes image under the twist $\psi_{s c}^{1 j}: G_{s c}^{1} \rightarrow G_{s c}^{j}$, then

$$
\left(g x_{s c}^{j} \sigma^{1}(g)^{-1}\right)^{\prime}=\left(g^{\prime} \sigma^{j}\left(g^{\prime}\right)^{-1}\right) \cdot\left(x_{s c}^{j}\right)^{\prime},
$$

and so mapping the image of $g^{\prime} \sigma^{j}\left(g^{\prime}\right)^{-1}$ in $T^{j}$ to the image of $g x_{s c}^{j} \sigma^{1}(g)^{-1}$ in $T^{1}$, for appropriate $g$, establishes the bijection. We thus identify $\mathcal{E}\left(T^{1}\right)$ with $\bigsqcup_{j} \mathcal{D}\left(T^{j}\right)$, 
and since

$$
\Phi_{\pi^{1}(1), \pi^{j}(w)}=\Phi_{\pi^{1}(1), \pi^{j}(1)} \Phi_{\pi^{j}(1), \pi^{j}(w)},
$$

the proof of Theorem 7.5 is now easily completed for discrete $\varphi$.

\section{Singular Elliptic Case}

A useful intermediate case is that where $\mathbf{G}$ is cuspidal and $\boldsymbol{\pi}$ is elliptic (in the sense of character theory). A standard representative $\varphi^{\prime}$ for $\varphi=\varphi(\pi)$ from [L2 factors through a discrete parameter for a cuspidal Levi subgroup ${ }^{L} M$ and so through ${ }^{L} T_{\mathbf{M}}$, where the maximal torus $\mathbf{T}_{\mathbf{M}}$ is compact modulo the center of $\mathbf{M}$. To follow the arguments of [S3], we construct a representative $\varphi$ factoring through ${ }^{L} T$, where $\mathbf{T}$ is compact modulo the center of $\mathbf{G}$. This new representative $\varphi$ will be of the form $\varphi(\mu, \lambda)$ of the last section, but now the regularity requirement on $\mu$ is dropped. We call such a $\varphi$ a limit of discrete parameters.

Recall that in the proof of the transfer theorem (see Section 14 of [S2]) we obtained, by transfer of a discrete parameter for an elliptic endoscopic group, a limit of discrete parameters $\varphi$. We then constructed a standard representative $\varphi^{\prime}$ (see Section 4.4 of [S3] ) using the fact that, in this setting, $\operatorname{Cent}\left(\varphi\left(\mathbb{C}^{\times}\right), G^{\vee}\right)$ is of type $\left(A_{1}\right)^{n}$, where $n$ is the number of positive roots outside the endoscopic group. We observe now that parameters with this property are also sufficient for inversion in the elliptic case.

Thus we start with standard representative $\varphi^{\prime}$ for tempered parameter $\varphi$, assuming $\varphi$ is relevant to $\mathbf{G}$ and that the attached extended $L$-packet contains an elliptic representation. We follow the setting of Section 5.4 of [S3, but now alter the notation slightly. Thus we now write $\varphi^{\prime}$ as discrete $\varphi_{M}(\mu, \lambda)$ relative to the cuspidal Levi group ${ }^{L} M$ in our usual manner $\underline{\mathrm{S} 2}$. As in 5.4 of [S3], a Levi group ${ }^{L} \widetilde{M}$ is then constructed using the Knapp-Zuckerman classification in the dual setting of Langlands (reviewed in 5.3 of [S3]); this dual construction allows us to handle all representations in an extended $L$-packet at once. The characters of these representations vanish on the regular semisimple conjugacy classes not meeting $\widetilde{\mathbf{M}}(\mathbb{R})$, and so we must have $\widetilde{\mathbf{M}}=\mathbf{G}$. Thus the representative $\varphi=\varphi(\mu, \lambda)$, written $\widetilde{\varphi}$ in [S3], is a limit of discrete parameters, and $\operatorname{Cent}\left(\varphi\left(\mathbb{C}^{\times}\right), G^{\vee}\right)$ is of type $\left(A_{1}\right)^{n}$ (see Proposition 5.4.2, loc. cit.). Notice that the data $(\mu, \lambda)$ comes from $\varphi^{\prime}$ as the discrete parameter for $\mathbf{M}$ (see Proposition 5.4.3, loc. cit.).

The representative $\varphi$ has the property that we may find a complete set of representatives $s_{s c}$ for $S_{\varphi}^{s c} /\left(S_{\varphi}^{s c}\right)^{0}$ in the maximal torus $\mathcal{T}_{s c}$. To check this we recall some steps in the proof of Theorem 5.4.4 of [S3]. First, Langlands' dual description of the $R$-group is used to construct representatives for $S_{\varphi^{\prime}} /\left(S_{\varphi^{\prime}}\right)^{0}$ as in the proof of (5.4.5) on p. 424. Clearly, these elements have analogues in $G_{s c}^{\vee}$, and these analogues then provide a complete set of representatives for $S_{\varphi^{\prime}}^{s c} /\left(S_{\varphi^{\prime}}^{s c}\right)^{0}$. Next $\varphi$ is defined as the conjugate of $\varphi^{\prime}$ given by the element $\mathbf{s}$ described on p. 423. The conjugates under $\mathbf{s}$ of the representatives chosen for $S_{\varphi^{\prime}}^{s c} /\left(S_{\varphi^{\prime}}^{s c}\right)^{0}$ all lie in $\mathcal{T}_{s c}$, and we thus obtain representatives $s_{s c}$ in $\mathcal{T}_{s c}$ for all elements of $S_{\varphi}^{s c} /\left(S_{\varphi}^{s c}\right)^{0}$, as desired. Notice also that the roots $\alpha_{i}^{\vee}$ of the proof are, with their negatives, exactly the roots of $\operatorname{Cent}\left(\varphi\left(\mathbb{C}^{\times}\right), G^{\vee}\right)$. For each representative $s_{s c}$, the roots $\alpha_{i}^{\vee}$ for which $\alpha_{i}^{\vee}\left(s_{s c}\right)=1$ are, with their negatives, exactly the roots of $\operatorname{Cent}\left(\varphi^{s}\left(\mathbb{C}^{\times}\right),\left(H^{s}\right)^{\vee}\right)$. If the parameter $\varphi^{s}$ attached to $s_{s c}$ is a discrete parameter for the attached endoscopic group, i.e., if $\alpha_{i}^{\vee}\left(s_{s c}\right)=-1$ for all $\alpha_{i}^{\vee}$, we recover the setting of the proof of transfer in 
Section 14 of [S2]; otherwise, $\varphi^{s}$ is also a limit of discrete parameters and we rely on coherent continuation.

Now suppose that $\eta \in X_{*}\left(\mathcal{T}_{s c}\right)$ is $G^{\vee}$-dominant and $G^{\vee}$-regular, so that $\varphi_{\eta}=$ $\varphi(\mu+\eta, \lambda)$ and $\varphi_{\eta}^{s}=\varphi\left(\mu^{s}+\eta, \lambda^{s}\right)$ are discrete parameters. Then the results of the last paragraph provide us with surjective homomorphisms $S_{\varphi_{\eta}}^{s c}=\mathbb{S}_{\varphi_{\eta}}^{s c} \rightarrow \mathbb{S}_{\varphi}^{s c}$ and $S_{\varphi_{\eta}}^{a d}=\mathbb{S}_{\varphi_{\eta}}^{a d} \rightarrow \mathbb{S}_{\varphi}^{a d}$, along with the evident commutative diagram. Suppose we attach to given representations $\boldsymbol{\pi}, \boldsymbol{\pi}^{\prime}$ (with parameter $\varphi$ ) the corresponding representations $\boldsymbol{\pi}(\eta), \boldsymbol{\pi}^{\prime}(\eta)$ with parameter $\boldsymbol{\varphi}_{\eta}$ described in Section 14 of [S2], i.e., $\boldsymbol{\pi}, \boldsymbol{\pi}^{\prime}$ are obtained from $\boldsymbol{\pi}(\eta), \boldsymbol{\pi}^{\prime}(\eta)$ respectively by coherent continuation. Then, by the result of the last section, for $s_{s c}$ in $S_{\varphi_{\eta}}^{s c}=\mathbb{S}_{\varphi_{\eta}}^{s c}$ we have the character

$$
s_{a d} \rightarrow \Phi\left(s_{s c}\right)=\frac{1}{\|\boldsymbol{\Delta}\|^{2}} \overline{\boldsymbol{\Delta}\left(\boldsymbol{\pi}(\eta)^{s}, \boldsymbol{\pi}(\eta)\right)} \boldsymbol{\Delta}\left(\boldsymbol{\pi}(\eta)^{s}, \boldsymbol{\pi}(\eta)^{\prime}\right)
$$

on $\mathbb{S}_{\varphi_{\eta}}^{a d}$. We argue by coherent continuation as in Section 14 of [S2] (see Section 5.4 of [S3] ), that the transfer factors $\boldsymbol{\Delta}\left(\boldsymbol{\pi}(\eta)^{s}, \boldsymbol{\pi}(\eta)\right), \boldsymbol{\Delta}\left(\boldsymbol{\pi}(\eta)^{s}, \boldsymbol{\pi}(\eta)^{\prime}\right)$ must coincide with $\boldsymbol{\Delta}\left(\boldsymbol{\pi}^{s}, \boldsymbol{\pi}\right), \boldsymbol{\Delta}\left(\boldsymbol{\pi}^{s}, \boldsymbol{\pi}^{\prime}\right)$ respectively, and thus $\Phi\left(s_{s c}\right)$ coincides with

$$
\frac{1}{\|\boldsymbol{\Delta}\|^{2}} \overline{\Delta\left(\boldsymbol{\pi}^{s}, \boldsymbol{\pi}\right)} \boldsymbol{\Delta}\left(\boldsymbol{\pi}^{s}, \boldsymbol{\pi}^{\prime}\right)
$$

Proof of Theorem 7.5 ( $\varphi$ elliptic). It is sufficient to show that $\Phi$ descends to a character on $\mathbb{S}_{\varphi}^{a d}$, and then that for fixed $\boldsymbol{\pi}$ we get exactly $\left|\mathbb{S}_{\varphi}^{a d}\right|$ distinct characters in this way.

To start, $\operatorname{Ker}\left(\mathbb{S}_{\varphi_{\eta}}^{a d} \rightarrow \mathbb{S}_{\varphi}^{a d}\right)=\operatorname{Ker}\left(S_{\varphi_{\eta}}^{a d} \rightarrow \mathbb{S}_{\varphi}^{a d}\right)$ is a subgroup of $\mathcal{T}_{a d}$ which we will describe shortly using arguments from the proof of Theorem 5.4.4 of [S3].

To show that $\Phi$ is trivial on $\operatorname{Ker}\left(\mathbb{S}_{\varphi_{\eta}}^{a d} \rightarrow \mathbb{S}_{\varphi}^{a d}\right)$, we proceed in two steps: if $\boldsymbol{\pi}, \boldsymbol{\pi}^{\prime}$ belong to the same group $G^{j}$, then we just use the argument for Lemma 5.4.19 in [S3, but if they belong to different groups, then we need a property of the $K$-group G that reflects the relevance of $\varphi$.

For this second step where the groups are different, we may assume that we are in the setting of the last section for the proof in the discrete case. We will further assume that $\boldsymbol{\pi}_{\eta}=\pi_{\eta}^{1}(1)$ and $\boldsymbol{\pi}_{\eta}^{\prime}=\pi_{\eta}^{j}(1)$, as we may easily reduce to this case. Suppose we transport the class of $x_{s c}^{j}$ to $T^{*}$ by $\psi^{1}$ to obtain an element, say $x$, of $H^{1}\left(\Gamma, T^{*}\right)$, and suppose that $t$ is an element of $S_{\varphi_{\eta}}^{a d}$, i.e., $t$ is an element of $\mathcal{T}_{a d}$ fixed by the Galois action of $\mathbf{T}$, and that $t$ lies in $\operatorname{Ker}\left(S_{\varphi_{\eta}}^{a d} \rightarrow \mathbb{S}_{\varphi}^{a d}\right)$. Then we have to show that

$$
\langle x, t\rangle=1 .
$$

Recall that $x=\left(u^{1}\right)^{-1} u^{j} \quad$ (Section 4), and each of $u^{1}, u^{j}$ define cocycles $u_{a d}^{1}, u_{a d}^{j}$ in the adjoint form $\mathcal{T}_{a d}$. We will check that $t$ is the image of an element $t_{s c}$ in $\operatorname{Ker}\left(S_{\varphi_{\eta}}^{s c} \rightarrow \mathbb{S}_{\varphi}^{s c}\right)$ under the projection $\mathcal{T}_{s c} \rightarrow \mathcal{T}_{\text {ad }}$ with the property that

$$
\left\langle u_{a d}^{1}, t_{s c}\right\rangle=\left\langle u_{a d}^{j}, t_{s c}\right\rangle
$$

Since $\langle x, t\rangle$ may be calculated as

$$
\left\langle x_{a d}, t_{s c}\right\rangle=\left\langle u_{a d}^{j}, t_{s c}\right\rangle /\left\langle u_{a d}^{1}, t_{s c}\right\rangle,
$$

we are then done with the second step. The proof of (5.4.6) in Theorem 5.4.4 of [S3] shows that we may construct the desired $t_{s c}$ and that it lies in the $\Gamma$-invariants in the center $Z\left(M_{s c}^{\vee}\right)$ of the Levi group $M_{s c}^{\vee}$ in $G_{s c}^{\vee}$. Then we calculate both $\left\langle u_{a d}^{1}, t_{s c}\right\rangle$ 
and $\left\langle u_{a d}^{j}, t_{s c}\right\rangle$ as $\zeta_{M}^{G}\left(t_{s c}\right)$ in the notation of Section 2 of [르, and so the result follows.

It remains now to count that we have the correct number of characters. We recall the discrete case from the last section and the exact sequence

$$
1 \rightarrow \mathcal{E}\left(T_{\mathbf{M}}^{1}\right) \rightarrow \mathbb{S}_{\varphi}^{a d} \rightarrow R_{\varphi} \rightarrow 1
$$

where $R_{\varphi}$ denotes Langlands' version of the $R$-group. From our explicit description of the extended $L$-packet in terms of coherently continued characters (see [S3] and Section 14 of [S2]), we see that we get exactly $\left|R_{\varphi}\right|$ such characters for each element $w$ of $\mathcal{E}\left(T_{\mathbf{M}}^{1}\right)=\bigsqcup_{k} \mathcal{D}\left(T_{\mathbf{M}}^{k}\right)$. Notice that because we have assumed that the $L$-packet contains an elliptic representation, we have the analogue of (5.4.15) of $\underline{S 3}$ for each of the $G^{j}$ comprising $\mathbf{G}$, but that our arguments for Theorem 7.5 apply equally as well with only the assumption $\widetilde{\mathbf{M}}=\mathbf{G}$.

\section{General tempered CASE}

To complete the proof of Theorem 7.5, it remains to consider the case that the cuspidal Levi group $\widetilde{\mathbf{M}}$ of the last section is proper. This is little more than an application of parabolic induction.

If $\varphi$ is regular, then $\widetilde{\mathbf{M}}=\mathbf{M}$, the $R$-group is trivial, and the map of $\mathcal{E}\left(T_{\mathbf{M}}^{1}\right)=\mathbb{S}_{\varphi_{M}}^{a d}$ into $\mathbb{S}_{\varphi}^{a d}$ is an isomorphism. We may lift this to an isomorphism $\mathbb{S}_{\varphi_{M}}^{s c} \rightarrow \mathbb{S}_{\varphi}^{s c}$, where again the superscript $s c$ for $M$ indicates the associated Levi group in $G_{s c}^{\vee}$. If $\boldsymbol{\pi}=I\left(\boldsymbol{\pi}^{\mathbf{M}}\right)$ and $\boldsymbol{\pi}^{\prime}=I\left(\left(\boldsymbol{\pi}^{\mathbf{M}}\right)^{\prime}\right)$, then write $\Phi^{M}$ for the character attached to $\boldsymbol{\pi}^{\mathbf{M}},\left(\boldsymbol{\pi}^{\mathbf{M}}\right)^{\prime}$ and $\Phi$ as usual. If $s_{s c}$ lies in $S_{\varphi_{M}}^{s c}$, then $\Phi^{M}\left(s_{s c}\right)=\Phi\left(s_{s c}\right)$ by the definition of transfer factors (Sections 8-10 of [S2]), and so Theorem 7.5 for regular $\varphi$ follows from the same result for the discrete $\varphi_{M}$.

In general, we still have irreducible induced representations, but now we invoke the results of the last section for $\widetilde{\mathbf{M}}$. We have again an isomorphism $\mathbb{S}_{\varphi_{\widetilde{M}}}^{s c} \rightarrow \mathbb{S}_{\varphi}^{s c}$. This is an immediate consequence of the version of Theorem 5.4.4 of [S3] in Section 9. If semisimple $s_{s c}$ lies in $S_{\varphi_{\widetilde{M}}}^{s c}$, then $\Phi^{\widetilde{M}}\left(s_{s c}\right)=\Phi\left(s_{s c}\right)$ by the construction of spectral transfer factors (see Section 14 of [S2]). This completes the proof of Theorem 7.5.

Remark 10.1. Observe that, as noted on p. 213 of [A1], we always have that the character $\zeta_{\mathbf{G}}$ is trivial on $\left(Z_{s c}^{\vee}\right)^{\Gamma} \cap\left(S_{\varphi}^{s c}\right)^{0}=Z_{s c}^{\vee} \cap\left(S_{\varphi}^{s c}\right)^{0}$.

\section{Structure of tempered $L$-PACKets}

In view of Theorem 7.5 it is tempting now to follow $\mathrm{S3}$ and simply identify the $L$-packet $\Pi$ attached to a tempered parameter $\varphi$ with the dual of $\mathbb{S}^{a d}=\mathbb{S}_{\varphi}^{a d}=$ $\pi_{0}\left(S_{\varphi}^{a d}\right)$, a finite sum of groups of order two, but that is not quite what is needed for global purposes [A1, at least when $\mathbf{G}$ is not of quasi-split type. Following [A1, and motivated by [AV], we have introduced the group $\mathbb{S}^{s c}=\mathbb{S}_{\varphi}^{s c}=\pi_{0}\left(S_{\varphi}^{s c}\right)$. This group is also abelian because it is a quotient of a subgroup of a maximal torus in $G_{s c}^{\vee}$. By construction, $S_{\varphi}^{s c}$ is an extension of $S_{\varphi}^{a d}$ by $Z_{s c}^{\vee}=Z\left(G_{s c}^{\vee}\right)$, and so $\mathbb{S}^{s c}$ is an extension of $\mathbb{S}^{a d}$ by the quotient of $Z_{s c}^{\vee}$ by $Z_{s c}^{\vee} \cap\left(S_{\varphi}^{s c}\right)^{0}$. The group $\mathbb{S}^{s c}$, while finite and abelian, is not necessarily a sum of groups of order two. The following small example is useful to keep in mind: for the $K$-group $\mathbf{S U}(2)=S U(2) \sqcup S U(2)$, the group $\mathbb{S}^{s c}$ is cyclic of order four when $\varphi$ is discrete. 
Recall that the character $\zeta_{\mathbf{G}}$ on $\left(Z_{s c}^{\vee}\right)^{\Gamma}$ is trivial on $\left(Z_{s c}^{\vee}\right)^{\Gamma} \cap\left(S_{\varphi}^{s c}\right)^{0}=Z_{s c}^{\vee} \cap\left(S_{\varphi}^{s c}\right)^{0}$ (see Remark 10.1). We then follow A1 and extend $\zeta_{\mathbf{G}}$ to a character on the subgroup

$$
Z_{s c}^{\vee} / Z_{s c}^{\vee} \cap\left(S_{\varphi}^{s c}\right)^{0}=\operatorname{Ker}\left(\mathbb{S}^{s c} \rightarrow \mathbb{S}^{a d}\right)
$$

of $\mathbb{S}^{s c}$ which we will also denote $\zeta_{\mathbf{G}}$. Now because $\mathbb{S}^{s c}$ is abelian we may make a further extension of $\zeta_{\mathbf{G}}$ to a character $\zeta$ on $\mathbb{S}^{s c}$ itself. In particular, if $\mathbf{G}$ is of quasisplit type, then we may take $\zeta$ to be the trivial character on $\mathbb{S}^{s c}$. On the other hand, $\zeta$ must be of order four in the case of $\mathbf{S U}(2)$. Also choose a member $\pi^{\text {base }}$ of the $L$-packet $\boldsymbol{\Pi}$ to serve as basepoint. There will be no harm in using the same notation for both a semisimple element $s_{s c}$ of $S^{s c}$ and its image in $\mathbb{S}^{s c}$. We define

$$
\left\langle s_{s c}, \pi^{\text {base }}\right\rangle=\zeta\left(s_{s c}\right) .
$$

We may now reinterpret Theorem 7.5 as:

\section{Corollary 11.1.}

(i) There is an unique extension of $\langle$,$\rangle to a pairing$

$$
\langle,\rangle: \mathbb{S}^{s c} \times \Pi \rightarrow \mathbb{C}^{\times}
$$

satisfying

$$
\left\langle s_{s c}, \boldsymbol{\pi}\right\rangle /\left\langle s_{s c}, \boldsymbol{\pi}^{\prime}\right\rangle=\boldsymbol{\Delta}\left(\boldsymbol{\pi}^{s}, \boldsymbol{\pi}\right) / \boldsymbol{\Delta}\left(\boldsymbol{\pi}^{s}, \boldsymbol{\pi}^{\prime}\right)
$$

for all $\boldsymbol{\pi}, \boldsymbol{\pi}^{\prime} \in \Pi, s_{s c} \in \mathbb{S}^{s c}$.

(ii) This pairing identifies $\Pi$ as the set of all characters on $\mathbb{S}^{s c}$ of the form $\zeta \otimes \omega$, where $\omega$ is a character trivial on the kernel of the projection $\mathbb{S}^{s c} \rightarrow$ $\mathbb{S}^{a d}$, i.e., as the set of all characters on $\mathbb{S}^{s c}$ whose restriction to $Z_{s c}^{\vee} /$ $Z_{s c}^{\vee} \cap\left(S_{\varphi}^{s c}\right)^{0}$ coincides with $\zeta_{\mathbf{G}}$.

We remark once again that $\boldsymbol{\Delta}\left(\boldsymbol{\pi}^{s}, \boldsymbol{\pi}\right) / \boldsymbol{\Delta}\left(\boldsymbol{\pi}^{s}, \boldsymbol{\pi}^{\prime}\right)$ coincides with the canonical spectral transfer factor $\boldsymbol{\Delta}\left(\boldsymbol{\pi}^{s}, \boldsymbol{\pi} ; \boldsymbol{\pi}^{s}, \boldsymbol{\pi}^{\prime}\right)$ of [S2] in the regular setting. In general, it is a sign. The following is also part of Arthur's conjecture in Section 3 of [A1].

\section{Corollary 11.2.}

$$
\boldsymbol{\Delta}\left(\boldsymbol{\pi}^{s}, \boldsymbol{\pi}\right)=\rho\left(\boldsymbol{\Delta}, s_{s c}\right)\left\langle s_{s c}, \boldsymbol{\pi}\right\rangle,
$$

where $\rho\left(\boldsymbol{\Delta}, s_{s c}\right)$ satisfies

$$
\rho\left(t \boldsymbol{\Delta}, z_{s c} s_{s c}\right)=t \rho\left(\boldsymbol{\Delta}, s_{s c}\right) \zeta_{\mathbf{G}}\left(z_{s c}\right)^{-1}
$$

for $t \in \mathbb{C}^{\times}$and $z_{s c} \in Z\left(G_{s c}^{\vee}\right)$.

Here, of course, we have an explicit formula:

$$
\rho\left(\boldsymbol{\Delta}, s_{s c}\right)=\zeta\left(s_{s c}\right)^{-1} \boldsymbol{\Delta}\left(\boldsymbol{\pi}^{s}, \boldsymbol{\pi}^{\text {base }}\right) .
$$

For the proof of the corollary, we observe that if $z_{s c} \in Z\left(G_{s c}^{\vee}\right)$ has image $z$ in $Z\left(G^{\vee}\right)$, then

$$
\boldsymbol{\Delta}\left(\boldsymbol{\pi}^{z s}, \boldsymbol{\pi}^{\text {base }}\right)=\boldsymbol{\Delta}\left(\boldsymbol{\pi}^{s}, \boldsymbol{\pi}^{\text {base }}\right) .
$$

This is because we have attached endoscopic data in such a way (see Section 7 ) that $\mathfrak{e}(z s)=\mathfrak{e}(s)$, and so the same $z$-pair will serve to construct both $\varphi^{z s}$ and $\varphi^{s}$. The construction then gives $\varphi^{z s}=\varphi^{s}$, so that we may take $\pi^{z s}=\boldsymbol{\pi}^{s}$, and the corollary follows. 
Remark 11.3 (Uniqueness of the pairing). The pairing depends of course on the base point $\boldsymbol{\pi}^{\text {base }}$ and the character $\zeta$ extending $\zeta_{\mathbf{G}}$. It does not, however, depend on the choice of representative $\varphi$ for the Langlands parameter provided we adjust $\zeta^{\prime}$ accordingly. To describe this more precisely, suppose $\varphi$ is replaced by $\varphi^{\prime}=$ $\operatorname{Int}(g) \circ \varphi$, where $g \in G^{\vee}$. Then if $g_{s c} \in G_{s c}^{\vee}$ has same image in $G_{a d}^{\vee}$ as $g$, we may use $g_{s c}$ to define a bijection $s_{s c} \rightarrow s_{s c}^{\prime}$ from semisimple elements in $S_{\varphi}^{s c}$ to those in $S_{\varphi^{\prime}}^{s c}$. This gives an isomorphism, also denoted $s_{s c} \rightarrow s_{s c}^{\prime}$, of $\mathbb{S}_{\varphi}^{s c}$ with $\mathbb{S}_{\varphi^{\prime}}^{s c}$, as well as an isomorphism $\zeta \rightarrow \zeta^{\prime}$ on characters such that $\zeta^{\prime}\left(s_{s c}^{\prime}\right)=\zeta\left(s_{s c}\right)$. As before, we write $s, s^{\prime}$ for the images of $s_{s c}, s_{s c}^{\prime}$ in $G^{\vee}$; Then $\mathfrak{e}\left(s^{\prime}\right)=g \cdot \mathfrak{e}(s)$, and we can take $\boldsymbol{\pi}^{s^{\prime}}$ for $\left(\boldsymbol{\pi}^{s}\right)^{\prime}$ in the notation of Section 2. If $\varphi$ is regular and $\boldsymbol{\pi}, \overline{\boldsymbol{\pi}}$ lie in $\boldsymbol{\Pi}$ we have

$$
\boldsymbol{\Delta}\left(\boldsymbol{\pi}^{s}, \boldsymbol{\pi}\right) / \boldsymbol{\Delta}\left(\boldsymbol{\pi}^{s}, \overline{\boldsymbol{\pi}}\right)=\boldsymbol{\Delta}\left(\boldsymbol{\pi}^{s}, \boldsymbol{\pi} ; \boldsymbol{\pi}^{s}, \overline{\boldsymbol{\pi}}\right)
$$

which, by Lemma 2.1(iii) in the setting of $K$-groups, coincides with

$$
\boldsymbol{\Delta}\left(\boldsymbol{\pi}^{s^{\prime}}, \boldsymbol{\pi} ; \boldsymbol{\pi}^{s^{\prime}}, \overline{\boldsymbol{\pi}}\right)=\boldsymbol{\Delta}\left(\boldsymbol{\pi}^{s^{\prime}}, \boldsymbol{\pi}\right) / \boldsymbol{\Delta}\left(\boldsymbol{\pi}^{s^{\prime}}, \overline{\boldsymbol{\pi}}\right)
$$

so that

$$
\boldsymbol{\Delta}\left(\boldsymbol{\pi}^{s}, \boldsymbol{\pi}\right) / \boldsymbol{\Delta}\left(\boldsymbol{\pi}^{s}, \overline{\boldsymbol{\pi}}\right)=\boldsymbol{\Delta}\left(\boldsymbol{\pi}^{s^{\prime}}, \boldsymbol{\pi}\right) / \boldsymbol{\Delta}\left(\boldsymbol{\pi}^{s^{\prime}}, \overline{\boldsymbol{\pi}}\right)
$$

in the regular case. We obtain the same result for all tempered $\varphi$ by applying the coherent continuation of Section 14 of [S2]. This and the definition of $\zeta$ allow us to conclude now that

$$
\left\langle s_{s c}, \boldsymbol{\pi}\right\rangle=\left\langle s_{s c}^{\prime}, \boldsymbol{\pi}\right\rangle
$$

for all $\pi \in \Pi$, semisimple $s_{s c} \in S_{\varphi}^{s c}$, and so our assertion is proved.

Next, write $\operatorname{Tr}\left(\boldsymbol{\Pi}, s_{s c}\right)$ for the endoscopic character

$$
\mathbf{f} \rightarrow \operatorname{St}-\operatorname{Tr} \boldsymbol{\pi}^{s}\left(\mathbf{f}^{s}\right)=\sum_{\boldsymbol{\pi} \in \boldsymbol{\Pi}} \boldsymbol{\Delta}\left(\boldsymbol{\pi}^{s}, \boldsymbol{\pi}\right) \operatorname{Tr} \boldsymbol{\pi}(\boldsymbol{f}) .
$$

This character depends, of course, on the normalization of the transfer factor $\boldsymbol{\Delta}$ (recall Remark 2.5 and Section 3). Notice that we may use the factor $\rho\left(\boldsymbol{\Delta}, s_{s c}\right)$ to shift the dependence:

$$
\operatorname{Tr}^{*}\left(\boldsymbol{\Pi}, s_{s c}\right)=\rho\left(\boldsymbol{\Delta}, s_{s c}\right)^{-1} \operatorname{Tr}\left(\boldsymbol{\Pi}, s_{s c}\right)=\sum_{\boldsymbol{\pi} \in \boldsymbol{\Pi}}\left\langle s_{s c}, \boldsymbol{\pi}\right\rangle \operatorname{Tr} \boldsymbol{\pi}
$$

depends instead on the basepoint $\boldsymbol{\pi}^{\text {base }}$ for $\boldsymbol{\Pi}$ and the (constrained) character $\zeta$ by which we pair $\pi^{\text {base }}$.

Lemma 11.4. Every tempered endoscopic character on $\mathbf{G}(\mathbb{R})$ is a multiple of some $\operatorname{Tr}^{*}\left(\boldsymbol{\Pi}, s_{s c}\right)$.

Proof. We have to show that the tempered endoscopic character

$$
S t-\operatorname{Tr} \boldsymbol{\pi}_{1}\left(\mathbf{f}_{1}\right)=\sum_{\boldsymbol{\pi} \in \boldsymbol{\Pi}} \boldsymbol{\Delta}\left(\boldsymbol{\pi}_{1}, \boldsymbol{\pi}\right) \operatorname{Tr} \boldsymbol{\pi}(\boldsymbol{f}),
$$

attached to a set of endoscopic data $\mathfrak{e}=\left(H, \mathcal{H}, s_{\mathfrak{e}}, \xi\right)$ and $z$-pair $\left(H_{1}, \xi_{1}\right)$, is a multiple of some $S t-\operatorname{Tr} \boldsymbol{\pi}^{s}\left(\mathbf{f}^{s}\right)$. In view of our analysis in Sections 2 and 3 we may replace $\mathfrak{e}$ by isomorphic data and $\left(\mathbf{H}_{1}, \xi_{1}\right)$ by another $z$-pair whenever needed, and by Remark 11.3 we may use any convenient representative for a Langlands parameter. As always, we use $\xi$ to identify $\mathcal{H}$ as a subgroup of ${ }^{L} G$. We may then assume parameter (representative) $\varphi_{1}$ for $\boldsymbol{\pi}_{1}$ has image in $\xi_{1}(\mathcal{H})$, so that the attached parameter $\varphi$ for $\pi$ has image in $\mathcal{H}$. We may assume the datum $s_{\mathfrak{e}}$ lies in $S_{\varphi}$, writing now $s$, and then adjust $s$ again by a central element to assume instead that it is the 
image of $s_{s c}$ in $S_{\varphi}^{s c}$. Thus $\mathcal{H}=\mathcal{H}^{s}$ since both groups are generated by the image of $\varphi$ together with the identity component of the same centralizer in $G^{\vee}$. We may also assume $H^{(s)}=H$, so that $\mathfrak{e}=\mathfrak{e}(s)$, and use $\left(\mathbf{H}_{1}, \xi_{1}\right)$ for $\left(\mathbf{H}^{s}, \xi^{s}\right)$. Now $\varphi_{1}$ coincides with $\varphi^{s}$, and the lemma follows.

Assume now, and for the rest of this paper, that the $K$-group $\mathbf{G}$ is of quasisplit type and that we use the normalization of transfer factors $\boldsymbol{\Delta}_{\text {geom }}$ and $\boldsymbol{\Delta}_{\text {spec }}$ attached to Whittaker data $(B, \lambda)$ for $\mathbf{G}$, writing instead $\boldsymbol{\Delta}_{\lambda}$ for each factor.

From Lemma 11.4, Corollary 14.1 of [S2, along with Section 5 above, it follows that

$$
\boldsymbol{\Delta}_{\lambda}\left(\boldsymbol{\pi}^{s}, \boldsymbol{\pi}\right)= \pm 1
$$

for all tempered $\pi$ and semisimple $s_{s c} \in S^{s c}$ with image $s$ in $S$. On the other hand, we may assume that the character $\zeta$ on $\mathbb{S}^{s c}$ is trivial. Then (ii) of Corollary 11.1 implies that $\left\langle s_{s c}, \boldsymbol{\pi}\right\rangle$ depends only on the image of $s_{s c}$ in $\mathbb{S}^{a d}$, a sum of groups of order two, so that we have also

$$
\left\langle s_{s c}, \boldsymbol{\pi}\right\rangle= \pm 1 .
$$

Theorem 11.5. Assume that the basepoint $\boldsymbol{\pi}^{\text {base }}$ for $\boldsymbol{\Pi}$ is generic relative to $(B, \lambda)$. Then

$$
\rho\left(\boldsymbol{\Delta}_{\lambda}, s_{s c}\right)=\boldsymbol{\Delta}_{\lambda}\left(\boldsymbol{\pi}^{s}, \boldsymbol{\pi}^{\text {base }}\right)=1
$$

for all semisimple $s_{s c} \in S^{s c}$.

Recall that by results of Kostant [K] and Vogan [V], every tempered $L$-packet for $\mathbf{G}$ contains generic representations, and within a packet we may always choose (a unique class of) representations generic relative to given Whittaker data $(B, \lambda)$ or, just as well, generic relative to any pair in the $G(\mathbb{R})$-conjugacy class of $(B, \lambda)$. We will assume from now that $\boldsymbol{\pi}^{\text {base }}$ is so chosen, and that $\zeta$ is trivial.

Corollary 11.6. The pairing of Corollary 11.1 is given by

$$
\left\langle s_{s c}, \boldsymbol{\pi}\right\rangle=\boldsymbol{\Delta}_{\lambda}\left(\boldsymbol{\pi}^{s}, \boldsymbol{\pi}\right)
$$

for all semisimple $s_{s c} \in S^{s c}$ and $\boldsymbol{\pi} \in \boldsymbol{\Pi}$.

Corollary 11.7. The endoscopic characters

$$
\operatorname{Tr}^{*}\left(\boldsymbol{\Pi}, s_{s c}\right)=\operatorname{Tr}\left(\boldsymbol{\Pi}, s_{s c}\right)=\sum_{\boldsymbol{\pi} \in \boldsymbol{\Pi}}\left\langle s_{s c}, \boldsymbol{\pi}\right\rangle \operatorname{Tr} \boldsymbol{\pi}
$$

are determined uniquely by (the $G(\mathbb{R})$-conjugacy class of) $(B, \lambda)$.

We prove Theorem 11.5 in Section 13.

Remark 11.8 (Remark on notation). We switch now to $\left(B^{*}, \lambda^{*}\right)$ for Whittaker data for the rest of the paper. We will not need explicitly our earlier use of $\lambda^{*}$ in data attached to the embeddings of an endoscopic $L$-group. Also we continue to use $\psi$ for an inner twist. An additive character on $\mathbb{R}$ will be denoted $\psi_{\mathbb{R}}$. Generally, $T$ will denote a (maximally) compact maximal torus and $T^{*}$ a maximally split one. 


\section{Some EXAMPLES}

Recall first that the spectral transfer factors are defined in terms of nondegenerate coherent continuation. This is not always the way an $L$-packet is presented. For example, if we examine the form of the discrete parameter $\varphi(\mu, \lambda)$ from Section 8 (here we have returned to our normal use for the notation $\lambda$ ) we see that we may remove the regularity requirement on $\mu$ entirely, and still obtain a well-defined representative for a tempered Langlands parameter $\varphi$, and thus also an attached $L$-packet provided $\boldsymbol{\varphi}$ is relevant to the given cuspidal $K$-group $\mathbf{G}$. We can, at the same time, form the set of distributions $\Phi\left(w^{-1} \mu, \lambda, w^{-1} \Psi\right)$, for any group $G$ comprising $\mathbf{G}$. Recall that $\mu$ is dominant with respect to the set $\Psi$ of $\mathcal{B}$-simple roots of $\mathcal{T} ; w$ is an element of the Weyl group of $\mathcal{T}$. Each distribution, a limit of discrete series, is either identically zero or a tempered irreducible character,. The labelling of the individual characters, i.e., the choice of $w$, depends of course on the map $\mathcal{T} \rightarrow T^{\vee}$, where $T$ is compact modulo the center of $G$, used to transport data, but the set so obtained does not.

In Theorem 4.3.2 of [S3] we showed that if $\varphi$ is relevant to $G$, then the nonzero characters among these limits form the (nonempty) $L$-packet attached to $\varphi$, and that all limits are zero if $\varphi$ is not relevant. The argument was by induction on the dimension of $G$, using the fact that the transfer identity allows coherent continuation, nondegenerate or not, while the proof of endoscopic transfer required only the nondegenerate case. The extension of Theorem 4.3.2 to $K$-groups is immediate. Assume $\boldsymbol{\varphi}$ is relevant to $\mathbf{G}$ and that $\boldsymbol{\Pi}$ is the extended $L$-packet attached to $\boldsymbol{\varphi}$. Then, according to Remark 11.3, there is no harm in using the (generally degenerate) parameter $\varphi(\mu, \lambda)$ to describe the pairing on $\boldsymbol{\Pi}$.

We observe now how to work directly with the parameter $\varphi(\mu, \lambda)$ for some particular representations considered by Carayol and Knapp [CK]. We will make use of these representations in the proof of Theorem 11.5. First we remark on the setting of [CK, and so assume the connected reductive algebraic group $G$ is simplyconnected, semisimple, cuspidal and quasi-split over $\mathbb{R}$. Then the parameter $\lambda$ in $\varphi(\mu, \lambda)$ is always zero and may be omitted. We consider $\varphi(\mu)$ for $\mu=0$, which is permitted since now $\iota \in X_{*}(\mathcal{T})$. The $K$-group machinery may be ignored since $\varphi(0)$ is relevant only to a quasi-split component of $\mathbf{G}$ which, as we check below, is unique. Notice that $S_{\varphi(0)}=S_{\varphi(0)}^{a d}$ is simply the group of fixed points in $G^{\vee}$ under conjugation by the element $\varphi(0)(1 \times \sigma)$ of ${ }^{L} G$.

For a slightly more general setting that is useful for endoscopy, and needed for the argument of the next section, we allow the connected (as algebraic group) cuspidal $G$ to be reductive, and consider those $\varphi(\mu, \lambda)$, for which

$$
\left\langle\mu, \alpha^{\vee}\right\rangle=0
$$

for all roots $\alpha^{\vee}$ of $\mathcal{T}$ in $G^{\vee}$. Again we assume $G$ quasi-split over $\mathbb{R}$, for otherwise $\varphi(\mu, \lambda)$ is not relevant to $G$. We will assume also that the derived group of $G$ is simply-connected or, equivalently, that the center of $G^{\vee}$ is connected, as this guarantees the existence of such parameters for $G$ and its elliptic endoscopic groups.

To show the existence, let $T$ be a maximal torus over $\mathbb{R}$ that is compact (anisotropic) modulo the center of $G$, and fix $\mathcal{T} \rightarrow T^{\vee}$ as usual to transport data; we will have a particular way of choosing this shortly. By assumption, the weight $\iota$ defines a (rational) character on $T_{\text {der }}(\mathbb{R})$. We take any extension of this character to a continuous character on $T(\mathbb{R})$ and write its Langlands parameter as $\varphi_{T}(\mu+\iota, \lambda)$. 
Then, by the congruence recalled in Section $8, \varphi(\mu, \lambda)$ is a well-defined parameter for $G$. It is clear also that $\left\langle\mu, \alpha^{\vee}\right\rangle=0$ for all roots $\alpha^{\vee}$ of $\mathcal{T}$ in $G^{\vee}$.

We now define distributions $\Phi\left(w^{-1} \mu, \lambda, w^{-1} \Psi\right)=\Phi\left(\mu, \lambda, w^{-1} \Psi\right)$. Turning to endoscopy, because $\mu^{s}=\mu-\mu^{* s}$, where $\left\langle\mu^{* s}, \alpha^{\vee}\right\rangle=0$ for all roots $\alpha^{\vee}$ of $\mathcal{T}^{s}$ in $\left(H^{s}\right)^{\vee}$ (see Section 11 of [S1], also Section 8 above), $\varphi^{s}=\varphi\left(\mu^{s}, \lambda^{s}\right)$ has the same property relative to the endoscopic group $H^{s}$. Notice that, as in the original case, we have $\operatorname{Cent}\left(\varphi\left(\mathbb{C}^{\times}\right), G^{\vee}\right)=G^{\vee}$, and so $\varphi, \varphi^{s}$ each factor through all cuspidal Levi groups, and $G$ must be quasi-split for relevance. The $L$-packet attached to one of these parameters then forms the set of constituents of a unitary minimal principal series representation.

We continue to ignore the $K$-group structure for now. We identify $G$ with its basic endoscopic group $G^{*}$ and take the inner twist $\psi$ to be the identity. We have fixed $\Gamma$-splitting $\operatorname{spl}_{G^{\vee}}=\left(\mathcal{B}, \mathcal{T},\left\{X_{\alpha^{\vee}}\right\}\right)$ of $G^{\vee}$, defining $\varphi(\mu)$ uniquely up to $\mathcal{T}$ conjugacy, and $\mathbb{R}$-splitting $s p l^{*}=\left(B^{*}, T^{*},\left\{X_{\alpha}\right\}\right)$ of $G$ for transport purposes, and $T$ is a maximal torus over $\mathbb{R}$ in $G$, compact modulo center. We are interested in $\mathbb{R}-$ opp splittings $\left(B, T,\left\{Y_{\alpha}\right\}\right)$. By this we mean $\sigma(B)$ is the Borel subgroup of $G$ opposite to $B$ relative to $T$ and $\sigma\left(Y_{\alpha}\right)=Y_{-\alpha}$ for each $B$-simple root $\alpha$. Here $Y_{-\alpha}$ is chosen to form a simple triple with $Y_{\alpha}, H_{\alpha}$. Notice that these splittings provide us exactly with the Borel subgroups $B$ containing $T$ for which all $B$-simple roots are noncompact (see Section 2 of $\mathbf{S} 4$ for a review of definitions in our setting).

Fix an $\mathbb{R}-o p p$ splitting $s p l=\left(B, T,\left\{Y_{\alpha}\right\}\right)$. If $s p l^{\prime}$ is also an $\mathbb{R}-o p p$ splitting, then $\left(s p l^{\prime}\right)^{w}=s p l$, where $w$ lies in $G_{s c}$, and we see easily that $\sigma(w)$ has the same property as $w$, and so $w_{\sigma}=\sigma(w) w^{-1}$ lies in the center $Z_{s c}$ of $G_{s c}$. Conversely, if $w_{\sigma}=\sigma(w) w^{-1}$ lies in $Z_{s c}$, then $(s p l)^{w^{-1}}$ is an $\mathbb{R}-o p p$ splitting. Thus the $G(\mathbb{R})$ conjugacy classes of $\mathbb{R}$ - opp splittings of $G$ are parametrized by $\operatorname{Ker}\left(H^{1}\left(\Gamma, Z_{s c}\right) \rightarrow\right.$ $\left.H^{1}(\Gamma, G)\right)$, where $H^{1}\left(\Gamma, Z_{s c}\right) \rightarrow H^{1}(\Gamma, G)$ is given by inclusion of $Z_{s c}$ in $G$. We will observe in Remark 12.3 that in the present setting this kernel is the entire group $H^{1}\left(\Gamma, Z_{s c}\right)$.

On the other hand, write $s p l$ as $\left(s p l^{*}\right)^{h}$, where the element $h$ of $G_{s c}$ acts by conjugation. Then we may use $h$ to produce $\mathcal{T} \rightarrow T^{\vee}$, as well as toral data including $T$. We thus attach the distributions $\Phi\left(\mu, \lambda, w^{-1} \Psi\right)$ to $\varphi(\mu, \lambda)$, where now we regard $\Psi$ as the $B$-simple roots of $T$, and we assume, without harm, that $w$ from the last paragraph normalizes $T$, and so acts as an element of the Weyl group of $T$. We associate $w_{\sigma}$ to $\Phi\left(\mu, \lambda, w^{-1} \Psi\right)$. Recall that for a general Weyl group element $w$, the distribution $\Phi\left(\mu, \lambda, w^{-1} \Psi\right)$ is nonzero if and only if $w^{-1} \Psi$ consists of noncompact roots, and so we also parametrize the nonzero distinct characters among the $\Phi\left(\mu, \lambda, w^{-1} \Psi\right)$ by $\operatorname{Ker}\left(H^{1}\left(\Gamma, Z_{s c}\right) \rightarrow H^{1}(\Gamma, G)\right)$, i.e. by $H^{1}\left(\Gamma, Z_{s c}\right)$. Thus the $L$-packet $\Pi$ attached to $\varphi(\mu, \lambda)$ consists of $\mid \operatorname{Ker}\left(H^{1}\left(\Gamma, Z_{s c}\right) \mid\right.$ representations.

Each of the representations in $\Pi$ is large (Theorem 6.2 of $[\mathrm{V}]$ ) and therefore generic $[\mathrm{K}, \mathrm{V}]$. Distinct (classes of) representations are evidently generic relative to distinct $G(\mathbb{R})$-conjugacy classes of Whittaker data. We wait to introduce Whittaker normalization of the transfer factors until after the proof of the next lemma. Instead, we simply take $\pi^{\text {base }}=\Phi(\mu, \lambda, \Psi)$, and assume that $\pi^{\text {base }}$ is paired as the trivial character. If $\Phi\left(\mu, \lambda, w^{-1} \Psi\right)$ is nonzero, write $\pi(w)$ for the class of representations with this character. We map the class of $w_{\sigma}$ in $H^{1}\left(\Gamma, Z_{s c}\right)$ to its image $w_{T}$ under $H^{1}\left(\Gamma, Z_{s c}\right) \rightarrow H^{1}\left(\Gamma, T_{s c}\right)$. Also, given a semisimple element $s_{a d}$ of $S_{\varphi(\mu, \lambda)}^{a d}$, we define $s_{T} \in\left(T_{a d}^{\vee}\right)^{\Gamma}$ in the usual manner: conjugate $s_{a d}$ to an element of $\mathcal{T}_{a d}$ and transport that element to an element $s_{T}$ of $T_{a d}^{\vee}$ fixed by the Galois action of $T$. 
The choice of conjugation does not matter because $w_{\sigma}$ is central (see the proof of Lemma 3.2.A of [LS1] for a similar argument). Denote the Tate-Nakayama pairing on $T_{s c}$ by $\langle,\rangle_{T}$. Then:

\section{Lemma 12.1.}

$$
\left\langle s_{s c}, \pi(w)\right\rangle=\left\langle s_{T}, w_{T}\right\rangle_{T}
$$

Proof. Divide the left side by $\left\langle s_{s c}, \pi(1)\right\rangle$ which, by assumption, is trivial. The result is, by Corollary 11.1 above, $\Delta\left(\pi^{s}, \pi(w)\right) / \Delta\left(\pi^{s}, \pi(1)\right)$, and so we are back to examining transfer factors. First we recall how to assemble suitable data.

First we conjugate $\varphi(\mu, \lambda)$ to a standard representative $\varphi^{\prime}$. The data for $\varphi^{\prime}$ must be of the form $\left(\mu, \lambda^{\prime}\right)$ relative to the minimal Levi group $T^{*}$, and so $\Pi$ consists of the constituents of a unitary minimal principal series representation. To present these constituents with nondegenerate data, we then pass to $\widetilde{\varphi}=\varphi_{\widetilde{M}}(\mu, \widetilde{\lambda})$ in the usual manner ([S3], Section 5). To simplify notation, we now write $M$ for $\widetilde{M}$. Thus each $\Phi\left(\mu, \lambda, w^{-1} \Psi\right)$ is parabolically induced from a similarly defined $\Phi_{M}\left(\mu, \widetilde{\lambda}, w_{M}^{-1} \Psi_{M}\right)$ for this cuspidal Levi group $M$ of type $\left(A_{1}\right)^{n}$.

Because the cocycle $w_{\sigma}$ is central, conjugation by $w$ defines an automorphism of $G(\mathbb{R})$. Multiplying by an element of $G(\mathbb{R})$, we may assume that $w$ defines an automorphism of $M(\mathbb{R})$ of the same kind $[\mathbf{S} 4$. From the descent properties of transfer factors [S2, we have only to examine the factors for $M$. We may continue coherently back into the chamber and assume that $\pi_{M}(1), \pi_{M}(w)$ are discrete series. We argue as in the proof of Lemma 3.2.A of [LS1] again to see that we may compute the pairing on the right in a fundamental maximal torus $T_{M}$ of $M$. Then we have only to recall from S2 how to compute $\Delta\left(\pi^{s}, \pi(w)\right) / \Delta\left(\pi^{s}, \pi(1)\right)$ in a discrete $L$-packet to complete the proof of the lemma.

We now use Whittaker normalization of the transfer factor. To specify a particular set $\left(B^{*}, \lambda^{*}\right)$ of Whittaker data, we fix the $\mathbb{R}$-splitting $s p l^{*}=\left(B^{*}, T^{*},\left\{X_{\alpha}\right\}\right)$ as usual, along with a nontrivial additive character $\psi_{\mathbb{R}}$ on $\mathbb{R}$. We then define $\lambda^{*}$ on a real point $u$ of the unipotent radical of $B^{*}$ as always: if $u=\exp x_{\alpha_{1}} X_{\alpha_{1}} \exp x_{\alpha_{2}} X_{\alpha_{2}} \ldots$ modulo commutators, where $\alpha_{1}, \alpha_{2}, \ldots$ is an enumeration of the $B^{*}$-simple roots, then $\lambda^{*}(u)=\psi_{\mathbb{R}}\left(x_{\alpha_{1}}+x_{\alpha_{2}}+\ldots\right)$. Recall that the transfer factor $\Delta_{\lambda^{*}}$ has one term depending on $\psi_{\mathbb{R}}$ and one on $s p l^{*}$, but the factor itself, i.e., the product, depends only on the resulting character $\lambda^{*}[\mathrm{KS}$ or, just as well, on the $G(\mathbb{R})$-conjugacy class of $\left(B^{*}, \lambda^{*}\right)$. Once we fix $\psi_{\mathbb{R}}$, we may identify the $G(\mathbb{R})$-conjugacy classes of data with the $G(\mathbb{R})$-conjugacy classes of $\mathbb{R}$-splittings of $G$.

Now we may choose an $\mathbb{R}-o p p$ splitting $s p l=\left(s p l^{*}\right)^{h}$ of $G$, and transfer dual data as above, so that the representation $\pi(1)$ with character $\Phi(\mu, \lambda, \Psi)$ is generic with respect to $\left(B^{*}, \lambda^{*}\right)$. Suppose $(s p l)^{w^{-1}}$ is another $\mathbb{R}-o p p$ splitting of $G$, where $w$ normalizes $T$. Then $\pi(w)$ is generic with respect to $\left(B^{*}, \lambda^{*}\right)^{w^{-1}}$, and, if we assume Theorem 11.5 for $\pi=\pi(1)$, we have

$$
\Delta_{\lambda^{*}}\left(\pi^{s}, \pi(w)\right)=\left\langle s_{s c}, \pi(w)\right\rangle=\left\langle s_{T}, w_{T}\right\rangle_{T}
$$

for all semisimple $s_{s c}$ in $S_{\varphi}^{s c}$. Here, as usual for a quasi-split group, we have designated the base point $\pi(1)$ to pair as the trivial character. The simple pairing on the right may be computed in terms of any maximal torus $T$ shared with the endoscopic group $H^{s}$, and so we will write just $\langle s, w\rangle$.

Inversion of endoscopic transfer now expands each character $\operatorname{Tr} \pi(w)=$ $\Phi\left(\mu, \lambda, w^{-1} \Psi\right)$ as: 
Lemma 12.2.

$$
\operatorname{Tr} \pi(w)(f)=\frac{1}{\left|H^{1}\left(\Gamma, Z_{s c}\right)\right|} \sum_{s_{s c}}\langle s, w\rangle S t-\operatorname{Tr} \pi^{s}\left(f^{s}\right) .
$$

Here $S t-\operatorname{Tr} \pi^{s}$ is the sum in $H^{s}$ of all characters $\Phi\left(\mu^{s}, \lambda^{s}, w_{s}^{-1} \Psi_{s}\right)$ attached to $\varphi^{s}=\varphi\left(\mu^{s}, \lambda^{s}\right)$. As we have observed in the proof of Lemma 12.1, this stable character $S t-\operatorname{Tr} \pi^{s}$ is a minimal principal series for $H^{s}$. Write $\varphi(\mu, \lambda)$ as $\varphi$. Then the summation $\sum_{s c}$ is over any set of semisimple elements $s_{s c}$ in $S_{\varphi}^{s c}$ whose images in $G_{a d}^{\vee}$ represent the components of the group of fixed points in $G_{a d}^{\vee}$ of conjugation by the element $\varphi(1 \times \sigma)$ of ${ }^{L} G$. For example, for $S U(2,1)$ we are free to use either $S U(2,1)$ or $U(1,1)$ as the endoscopic group contributing the one term on the right side.

Notice that if we use a general normalization of transfer factors in the inversion, then we may retain the right side of this formula as long as we replace the left side by

$$
\Delta\left(\pi^{s}, \pi(1)\right) \operatorname{Tr} \pi(w)(f) .
$$

Recall that $\Delta\left(\pi^{s}, \pi(1)\right) /\left|\Delta\left(\pi^{s}, \pi(1)\right)\right|$ is a fourth root of unity [S2].

Remark 12.3. We have yet to check that $H^{1}\left(\Gamma, Z_{s c}\right) \rightarrow H^{1}(\Gamma, G)$ is the trivial map. Here, with Remark 12.5 in mind, we allow $G$ to be any connected reductive quasisplit group. This is presumably well-known and can be verified in several ways. It is enough to check that $H^{1}(\Gamma, Z) \rightarrow H^{1}(\Gamma, G)$ is trivial under the assumption that $G$ is simply-connected and semisimple. By a familiar descent to a cuspidal Levi group, we may also assume $G$ cuspidal. We reduce immediately to absolutely simple groups. We have seen that the cardinality of the $L$-packet $\Pi$ attached to $\varphi(\mu, \lambda)$ is $\left|\operatorname{Ker}\left(H^{1}(\Gamma, Z) \rightarrow H^{1}(\Gamma, G)\right)\right|$. On the other hand, the cardinality of $\Pi$ is also the same as the cardinality of the $R$-group for the standard representative $\varphi^{\prime}$. The cardinality of this group is listed in the fifth column of Table 5.1 of [CK] . In each case, that number coincides with the cardinality of $H^{1}(\Gamma, Z)$.

Alternatively, to check directly that the map $H^{1}(\Gamma, Z) \rightarrow H^{1}(\Gamma, G)$ is trivial when $G$ is simple, simply-connected, cuspidal and quasi-split, we have only to notice the following.

Lemma 12.4. In this setting, the image of $H^{1}(\Gamma, Z)$ in $H^{1}(\Gamma, T)$ lies in the set $\mathcal{D}(T)$.

Proof. We identify $H^{1}(\Gamma, T)$ with

$$
H^{-1}\left(\Gamma, X_{*}(T)\right)=X_{*}(T) / 2 X_{*}(T),
$$

by Tate-Nakayama duality. The image of $H^{1}(\Gamma, Z)$ is then the image of

$$
H^{-2}\left(\Gamma, X_{*}\left(T_{a d}\right) / X_{*}(T)\right) \rightarrow H^{-1}\left(\Gamma, X_{*}(T)\right),
$$

where $T_{a d}=T / Z$, and so consists of the cosets represented by twice a coweight. We may assume the simple roots are noncompact, and then the coset of a sum of orthogonal simple coroots lies in $\mathcal{D}(T)$ (see, for example, calculations in Sections 2, 3 of [S6]). Suppose $H^{1}(\Gamma, Z) \neq 1$. Then in each case we observe from the tables in B a coweight $\lambda^{\vee}$ for which the coset of $2 \lambda^{\vee}$ is (i) nontrivial and (ii) represented by a sum of orthogonal simple coroots, and that finishes the proof except for $D_{2 n}$. For $D_{2 n}$, we find a second (and third) such coweight giving a distinct nontrivial coset, and that is enough to finish the proof. 
Remark 12.5. Let $\mathbf{G}=\bigsqcup_{j} G^{j}$ be a $K$-group, with $G^{1}$ quasi-split over $\mathbb{R}$ and $\psi^{1}$ : $G^{1} \rightarrow G^{*}$ defined over $\mathbb{R}$. Then if $G^{j}$ is also quasi-split over $\mathbb{R}$ there is an inner automorphism Int $y_{s c}$ such that Int $y_{s c} \circ \psi^{j}$ is an $\mathbb{R}$-isomophism, and so the cocycle $u^{j}$ of Section 5 provides us with a cocycle $y_{s c} u^{j} \sigma\left(y_{s c}\right)^{-1}$ with values in $Z_{s c}^{*}$. By the $K$-group construction, the image in $H^{1}\left(\Gamma, G^{*}\right)$ of this cocycle is nontrivial if $j \neq 1$. According to Remark 12.3 that is impossible, and so we conclude:

Lemma 12.6. The quasi-split component of a K-group of quasi-split type is unique.

In some cases, such as $G^{1}=S U(n, n)$, this is the only component determined uniquely by its isomorphism class; Lemma 12.4 fails for all other inner forms of $S U(n, n)$. On the other hand, for $G^{1}=S U(n+1, n)$ every component is uniquely determined by its isomorphism class, and every inner form of $G^{1}$ appears in the $K$-group.

\section{Proof of Theorem 11.5}

Consider now any $K$-group $\mathbf{G}$ of quasi-split type, and denote by $\boldsymbol{\Delta}_{\lambda^{*}}$ the spectral transfer factor attached to the Whittaker data $\left(B^{*}, \lambda^{*}\right)$. Lemma 12.6 allows us to ignore the $K$-group structure. To save notation we also assume that quasi-split $G=G^{j}$ coincides with $G^{*}$, and that the inner twist $\psi^{j}$ is the identity. We will also fix throughout an additive character $\psi_{\mathbb{R}}$ on $\mathbb{R}$, and use it with our chosen splitting $s p l^{*}=\left(B^{*}, T^{*},\left\{X_{\alpha}\right\}\right)$ of $G$ to specify the Whittaker data $\left(B^{*}, \lambda^{*}\right)$. We then specify other Whittaker data by adjusting only the splitting.

First we reduce to the case that $G_{d e r}$ is simply-connected, so that the representations of the last section are available for the cuspidal Levi groups in $G$. Recall that for many problems in ordinary endoscopy we may pass without harm to a $z$-extension $G_{1}$ of a given connected reductive group $G$ [L1, LS1, and that the derived group of $G_{1}$ is simply-connected. Theorem 11.5 asserts that

$$
\Delta_{\lambda^{*}}\left(\pi^{s}, \pi\right)=1
$$

if $\pi$ is generic relative to $\left(B^{*}, \lambda^{*}\right)$, where $s$ denotes the image in $G^{\vee}$ of given $s_{s c}$ in $S^{s c}$. We regard $\pi$ as a generic representation of $G_{1}(\mathbb{R})$ which transforms trivially under the central kernel $Z_{1}(\mathbb{R})$ of the surjective homomorphism $G_{1}(\mathbb{R}) \rightarrow G(\mathbb{R})$, and its Langlands parameter $\varphi$ as a homomorphism into ${ }^{L} G_{1}$ with image in the subgroup ${ }^{L} G$. Then $s_{s c}, s$ are unchanged, and the $z$-extension $H^{s}$ may be chosen as in Section 4.4 of [LS1]. Recall from Section 3 that the particular choice of $z$-extension does not affect our results. We lift $\left(B^{*}, \lambda^{*}\right)$ and $\Delta_{\lambda^{*}}\left(\pi^{s}, \pi\right)$ to $G_{1}$, observing term by term, including the epsilon factor, that we again have the Whittaker normalization. The reduction step now follows.

Next assume also that $G$ is cuspidal, and the parameter $\varphi=\varphi(\mu, \lambda)$ for $\pi$ is discrete. Choose toral data with $T$ compact modulo center for which we may write $\pi=\pi(1)=\Phi(\mu, \lambda, \Psi)$. Recall that we can do this by attaching to $\pi$ a $\mathbb{R}-$ opp splitting as in Section 12. Now use $\iota=\iota \Psi$ to form a parameter $\varphi_{0}=\varphi\left(\mu_{0}, \lambda_{0}\right)$ with $\left\langle\mu_{0}, \alpha^{\vee}\right\rangle=0$ for all roots $\alpha$ in $G$, also as in Section 12. Set $\pi_{0}=\pi_{0}(1)=$ $\Phi\left(\mu_{0}, \lambda_{0}, \Psi\right)$. Then $\left(\mu-\mu_{0}, \lambda-\lambda_{0}\right)$ defines a character $\Lambda\left(\mu-\mu_{0}, \lambda-\lambda_{0}\right)$ on $T(\mathbb{R})$ and provides the necessary data for coherent continuation from $\pi$ to $\pi_{0}$ (see Section 5 of [SV]). Here the role of $\lambda-\lambda_{0}$ is to provide a needed shift in central character. Recall that in the present setting both $G_{d e r}(\mathbb{R})$ and $T_{\text {der }}(\mathbb{R})$ are connected, while $T(\mathbb{R})=$ $T_{\text {der }}(\mathbb{R}) Z(\mathbb{R})$ and the various $\Phi(w \mu, \lambda, w \Psi)$ are supported on $G_{\text {der }}(\mathbb{R}) Z(\mathbb{R})$. Because 
$G_{d e r}(\mathbb{C})$ is connected and simply-connected we may easily find a highest $T(\mathbb{C})$ weight that restricts to $\Lambda\left(\mu-\mu_{0}, \lambda-\lambda_{0}\right)$. We also have characters $\Lambda\left(w \mu-\mu_{0}, \lambda-\lambda_{0}\right)$, thus allowing a uniform continuation of the entire extended $L$-packet of $\pi$, which we abbreviate as the continuation of $\varphi$ to $\varphi_{0}$. Recall the transfer identity for $\left(\pi^{s}, \pi\right)$ at the $K$-group level:

$$
S t-\operatorname{Tr} \boldsymbol{\pi}^{s}\left(\mathbf{f}^{s}\right)=\sum_{\boldsymbol{\pi}^{\prime}, \text { temp }} \boldsymbol{\Delta}_{\lambda^{*}}\left(\boldsymbol{\pi}^{s}, \boldsymbol{\pi}^{\prime}\right) \operatorname{Tr} \boldsymbol{\pi}^{\prime}(\mathbf{f}) .
$$

The representations $\boldsymbol{\pi}^{\prime}$ contributing nontrivially to the right side are the representations $\pi(w)$ of the quasi-split form on which we are working, as well as some representations on other forms if $\mathcal{D}(T)$ is a proper subset of $\mathcal{E}(T)$. The latter representations contribute nothing after coherent continuation to $\varphi_{0}$ is applied to both sides of the transfer identity (more precisely, on the left, the continuation is to $\varphi_{0}^{s}$, as below), and so we may as well work exclusively with the identity

$$
S t-\operatorname{Tr} \pi^{s}\left(f^{s}\right)=\Delta_{\lambda^{*}}\left(\pi^{s}, \pi\right) \operatorname{Tr} \pi(f)+\sum_{w \neq 1} \Delta_{\lambda^{*}}\left(\pi^{s}, \pi(w)\right) \operatorname{Tr} \pi(w)(f)
$$

on $G(\mathbb{R})$, where the summation is over $w$ in $\mathcal{D}(T)$. The element $s_{s c}$ serves for both $\varphi$ and $\varphi_{0}$, and $\varphi^{s}=\varphi\left(\mu^{s}, \lambda^{s}\right)$ continues to $\varphi_{0}^{s}=\varphi\left(\mu_{0}^{s}, \lambda_{0}^{s}\right)$, where $\mu_{0}^{s}=\mu_{0}-\mu^{* s}$, $\lambda_{0}^{s}=\lambda_{0}-\lambda^{* s}$, as $\varphi$ continues to $\varphi_{0}$. The transfer identity then becomes

$$
S t-\operatorname{Tr} \pi_{0}^{s}\left(f^{s}\right)=\Delta_{\lambda^{*}}\left(\pi^{s}, \pi\right) \operatorname{Tr} \pi_{0}(f)+\sum_{w \neq 1} \Delta_{\lambda^{*}}\left(\pi^{s}, \pi(w)\right) \operatorname{Tr} \pi_{0}(w)(f),
$$

where now only those $w$ in the image of $H^{1}\left(\Gamma, Z_{s c}\right)$ contribute nontrivially to the summation on the right. But the coefficients on the right are, by the transfer theorem, $\Delta_{\lambda^{*}}\left(\pi_{0}^{s}, \pi_{0}\right)$ and $\Delta_{\lambda^{*}}\left(\pi_{0}^{s}, \pi_{0}(w)\right)$. Thus to prove Theorem 11.5 for the pair $\left(\pi^{s}, \pi\right)$ it is sufficient to check that

$$
\Delta_{\lambda^{*}}\left(\pi_{0}^{s}, \pi_{0}\right)=1
$$

Our assumption on the toral data or, more specifically on $T^{*} \rightarrow T$, is that $\pi$, generic relative to $\left(B^{*}, \lambda^{*}\right)$, is the member of the packet designated $\pi(1)=$ $\Phi(\mu, \lambda, \Psi)$. Since $\pi_{0}=\pi_{0}(1)=\Phi\left(\mu_{0}, \lambda_{0}, \Psi\right)$, we have that $\pi_{0}$ is generic relative to the same $\left(B^{*}, \lambda^{*}\right)$. This property for our coherent continuation (to the wall) follows easily from the arguments of Section 4.6 of $[\mathrm{K}]$.

Thus it remains to examine the identity

$$
S t-\operatorname{Tr} \pi_{0}^{s}\left(f^{s}\right)=\sum_{w} \Delta_{\lambda^{*}}\left(\pi_{0}^{s}, \pi_{0}(w)\right) \operatorname{Tr} \pi_{0}(w)(f),
$$

where the summation is over all $w$ in the image of $H^{1}\left(\Gamma, Z_{s c}\right)$. We may of course rewrite the right side as

$$
\Delta_{\lambda^{*}}\left(\pi_{0}^{s}, \pi_{0}\right) \sum_{w}\langle s, w\rangle \operatorname{Tr} \pi_{0}(w)(f),
$$

but that does not concern us here. The stable trace on the left is the character of a minimal principal series representation of the elliptic endoscopic group $H^{s}(\mathbb{R})$. On the right, we then have characters that are parabolically induced from a cuspidal Levi group $M$ with the property that none of its roots are from $H^{s}$. Thus $M$ is of type $\left(A_{1}\right)^{r}$, where $r$ is the number of positive roots in $M$. We may arrange that $\alpha_{1}, \alpha_{2}, \ldots, \alpha_{r}$ is a superorthogonal set of $B^{*}$-positive real roots of $T^{*}$ such that conjugation by $t \in M$ performs a sequence of standard inverse Cayley transforms 
with respect to these roots, mapping $T^{*}$ to a fundamental maximal torus $T_{M}$ in $M$. Thus $s p l^{*},\left(s p l^{*}\right)^{t}$ provide an $\mathbb{R}$-splitting and an $\mathbb{R}-o p p$ splitting for $M$. We now descend to $M: \pi_{0}$ is obtained by parabolic induction from a representation $\pi_{0, M}$ generic relative to the attached data for $M$, and

$$
\Delta_{\lambda^{*}}\left(\pi_{0}^{s}, \pi_{0}\right)=\Delta_{\lambda^{*}}\left(\pi_{0, M}^{s}, \pi_{0, M}\right) .
$$

We prove this last assertion by first recalling $G$-regular descent for the pairs $\left(H^{s}, G\right),\left(M_{1}, M\right)$ from Section 12 of [S2] and then applying (nondegenerate) coherent continuation as in Section 14 of [S2]. Here of course $M_{1}$ is simply the chosen maximally split torus in $H^{s}$, and by definition the epsilon factors in the Whittaker normalizations relative to $G$ and $M$ are the same. Thus we have only to prove

$$
\Delta_{\lambda^{*}}\left(\pi_{0, M}^{s}, \pi_{0, M}\right)=1 .
$$

Since $M$ is of type $\left(A_{1}\right)^{r}$, we can calculate concretely, starting with discrete series. With the standard (internal) choices we get

$$
\Delta_{\lambda^{*}}\left(\pi_{0, M}^{s}, \pi_{0, M}\right)=[(-i)(-1)(-i)(1)]^{r}=1,
$$

and so this step is done.

The last step in the proof is to drop the assumption that $G$ is cuspidal and the parameter $\varphi$ is discrete. Notice that in the argument of the last paragraph we may replace $G$ by a cuspidal Levi subgroup. Now to deal with a general tempered $L$ packet we combine descent to a cuspidal Levi subgroup with a coherent continuation as in Section 14 of S2] (see also Section 10 above). This finishes our proof of Theorem 11.5.

For each tempered $L$-packet $\Pi$ attached to a $K$-group of quasi-split type, we have now established the pairing

$$
\left\langle s_{s c}, \boldsymbol{\pi}\right\rangle=\boldsymbol{\Delta}_{\lambda^{*}}\left(\boldsymbol{\pi}^{s}, \boldsymbol{\pi}\right),
$$

for semisimple $s_{s c} \in S^{s c}, \boldsymbol{\pi} \in \Pi$. The dependence on $s_{s c}$ is through its image in the component group $\mathbb{S}^{a d}$, and we have a dual pairing of $\mathbb{S}^{a d}$ and $\boldsymbol{\Pi}$. The pairing of course depends on the Whittaker data $\left(B^{*}, \lambda^{*}\right)$ or, more precisely, on the $G(\mathbb{R})$ conjugacy class of $\left(B^{*}, \lambda^{*}\right)$.

Our final remark concerns a change in the $G(\mathbb{R})$-conjugacy class of $\left(B^{*}, \lambda^{*}\right)$. Write another set $\left(B^{* *}, \lambda^{* *}\right)$ of Whittaker data as $\left(B^{*}, \lambda^{*}\right)^{w^{-1}}$, where $w_{\sigma}=\sigma(w) w^{-1}$ lies in $Z_{s c}$, and define $\langle s, w\rangle$ as before (Section 12). This sign may be computed using either $H^{1}\left(Z_{s c}\right)$ or its image in $H^{1}(Z)$, so that it is constant on $G(\mathbb{R})$-conjugacy classes of data.

Lemma 13.1. For all semisimple $s_{s c} \in S^{s c}, \boldsymbol{\pi} \in \Pi$, we have

$$
\boldsymbol{\Delta}_{\lambda^{* *}}\left(\boldsymbol{\pi}^{s}, \boldsymbol{\pi}\right)=\langle s, w\rangle \boldsymbol{\Delta}_{\lambda^{*}}\left(\boldsymbol{\pi}^{s}, \boldsymbol{\pi}\right) .
$$

Proof. We can argue exactly the same way as for the geometric factors [KS]. Both transfer factors $\boldsymbol{\Delta}_{\lambda^{* *}}, \boldsymbol{\Delta}_{\lambda^{*}}$ are modifications of a standard factor $\boldsymbol{\Delta}_{0}$ by an epsilon factor. We can use the same epsilon factor by fixing the additive character $\psi_{\mathbb{R}}$, and change instead the $\mathbb{R}$-splitting used to define the term $\boldsymbol{\Delta}_{I}$ of $\boldsymbol{\Delta}_{0}$. Then we are back to the results in Section 3.2 of [LS1, and the lemma is proved.

Alternatively, since $\boldsymbol{\Delta}_{\lambda^{* *}}, \boldsymbol{\Delta}_{\lambda^{*}}$ are transfer factors, they differ by a constant (for given $s_{s c}$ ). We may thus check the result on a single tempered $L$-packet. We arrange that $\boldsymbol{\pi}^{s}$ belongs to the minimal principal series, and then the result is clear by a familiar reduction to $S L(2)$. 


\section{REFERENCES}

[A] Arthur, J., Problems for real groups, Contemp. Math., vol. 472 (2008), 39-62.

[A1] Arthur, J., A note on L-packets, Pure and Appl. Math. Quart., Coates vol. (2006), 199-217. MR 2217572 (2006k:22014)

[A2] Arthur, J., On the transfer of distributions: weighted orbital integrals, Duke Math. J., vol. 99 (1999), 209-283. MR1708030 (2000i:22023)

[ABV] Adams, J., D. Barbasch and D. Vogan, The Langlands Classification and Irreducible Characters for Real Reductive Groups. Birkhäuser, Boston, 1992. MR.1162533 (93j:22001)

[AJ] Adams, J. and J. Johnson, Endoscopic groups and packets of nontempered representations, Compositio Math., vol. 64 (1987), 271-309. MR918414 (89h:22022)

[AV] Adams, J. and D. Vogan, L-groups, projective representations, and the Langlands classification, Amer. J. Math., vol. 114 (1992), 45-138. MR.1147719 (93c:22021)

[B] Bourbaki, N., Groupes et algebres de Lie, Ch. 4, 5 et 6, Hermann, Paris, 1968. MR0240238 (39:1590)

[CK] Carayol, H. and A. Knapp, Limits of discrete series with infinitesimal character zero, Trans. Amer. Math Soc., vol. 359 (2007), 5611-5651. MR2327045 (2008f:22011)

[K] Kostant, B., On Whittaker vectors and representation theory, Invent. Math., vol. 48 (1978), 101-184. MR507800 (80b:22020)

[K1] Kottwitz, R., Stable trace formula: elliptic singular terms, Math. Ann., vol. 275 (1986), 365-399. MR 858284 (88d:22027)

[K2] Kottwitz, R., Sign changes in harmonic analysis on reductive groups, Trans. Am. Math. Soc., vol. 278 (1983), 289-297. MR697075 (84i:22012)

[KS] Kottwitz, R. and D. Shelstad, Foundations of Twisted Endoscopy, Asterisque, vol. 255, 1999 MR 1687096 (2000k:22024)

[KZ] Knapp, A. and G. Zuckerman, Classification of irreducible tempered representations of semisimple Lie groups, Proc. Nat. Acad. Sci. USA, vol. 73 (1976), 2178-2180. MR0460545 $(57: 538)$

[L1] Langlands, R., Stable conjugacy: definitions and lemmas, Canad. J. Math., vol. 31 (1979), 700-725. MR.540901 (82j:10054)

[L2] Langlands, R., On the classification of irreducible representations of real algebraic groups, in Representation Theory and Harmonic Analysis on Semisimple Lie Groups, AMS Math Surveys and Monographs, vol. 31, 1989, 101-170. MR.1011897(91e:22017)

[LS1] Langlands, R. and D. Shelstad, On the definition of transfer factors, Math. Ann., vol. 278 (1987), 219-271. MR.909227 (89c:11172)

[S1] Shelstad, D., Tempered endoscopy for real groups I: geometric transfer with canonical factors, Contemp. Math., vol. 472 (2008), 215-248.

[S2] Shelstad, D., Tempered endoscopy for real groups II: spectral transfer factors, in Automorphic Forms and the Langlands Program, International Press, 243-282 (in press).

[S3] Shelstad, D., L-indistinguishability for real groups, Math. Ann., vol. 259 (1982), 385-430. MR661206 (84c:22017)

[S4] Shelstad, D., Characters and inner forms of a quasi-split group over R, Compositio Math., vol. 39 (1979), 11-45. MR539000 (80m:22023)

[S5] Shelstad, D., Some character relations for real reductive algebraic groups, thesis.

[S6] Shelstad, D., Orbital integrals and a family of groups attached to a real reductive group, Ann. Scient. Ec. Norm. Sup. vol. 12, (1979), 1-31. MR532374 (81k:22014)

[S7] Shelstad, D., Examples in endoscopy for real groups (notes for BIRS summer school, Aug. 08), preprint.

[SV] Speh, B. and D. Vogan Reducibility of generalized principal series representations, Acta Math., vol. 145 (1980), 227-299. MR590291 (82c:22018)

[V] Vogan, D., Gelfand-Kirillov dimension for Harish Chandra modules, Invent. Math., vol. 48 (1978), 75-98. MR0506503(58:22205)

Department of Mathematics, Rutgers University, Newark, New Jersey 07102

E-mail address: shelstad@rutgers.edu 